\title{
Effects of the Submesoscale on the Potential Vorticity Budget of Ocean Mode Waters
}

\author{
JACOB O. WenEGRAT AND LEIF N. ThOMAS \\ Department of Earth System Science, Stanford University, Stanford, California \\ JONATHAN GULA \\ Université de Brest, CNRS, IRD, Ifremer, Laboratoire d'Océanographie \\ Physique et Spatiale, IUEM, Brest, France \\ JAMES C. MCWILLIAMS \\ Department of Atmospheric and Oceanic Sciences, University of California, \\ Los Angeles, Los Angeles, California
}

(Manuscript received 24 October 2017, in final form 12 July 2018)

\begin{abstract}
Nonconservative processes change the potential vorticity (PV) of the upper ocean and, later, through the subduction of surface waters into the interior, affect the general ocean circulation. Here we focus on how boundary layer turbulence, in the presence of submesoscale horizontal buoyancy gradients, generates a source of potential vorticity at the ocean surface through a balance known as the turbulent thermal wind. This source of PV injection at the submesoscale can be of similar magnitude to PV fluxes from the wind and surface buoyancy fluxes, and hence can lead to a net injection of PV onto outcropped isopycnals even during periods of surface buoyancy loss. The significance of these dynamics is illustrated using a high-resolution realistic model of the North Atlantic Subtropical Mode Water (Eighteen Degree Water), where it is demonstrated that injection of PV at the submesoscale reduces the rate of mode water PV removal by a factor of $\sim 2$ and shortens the annual period of mode water formation by $\sim 3$ weeks, relative to air-sea fluxes alone. Submesoscale processes thus provide a direct link between small-scale boundary layer turbulence and the gyrescale circulation, through their effect on mode water formation, with implications for understanding the variability and biogeochemical properties of ocean mode waters globally.
\end{abstract}

\section{Introduction}

Submesoscale processes, characterized by horizontal scales of $0.1-10 \mathrm{~km}$ and Rossby and Richardson numbers of $O(1)$, modify the dynamics and properties of the upper ocean in a wide range of fundamental ways (as reviewed in Thomas et al. 2008; McWilliams 2016). In this manuscript we focus on how boundary layer turbulence at submesoscale buoyancy fronts results in a nonadvective source of potential vorticity $(\mathrm{PV})$ at the ocean surface. The relevant dynamics can be understood in the context of recent work on ageostrophic flows at fronts, through a balance called the turbulent thermal wind (TTW), a three-term balance between Coriolis acceleration, the baroclinic pressure gradient force, and

\footnotetext{
Corresponding author: Jacob O. Wenegrat, jwenegrat@stanford. edu
}

the vertical momentum flux divergence (McWilliams et al. 2015; Wenegrat and McPhaden 2016). The PV fluxes that arise through the TTW circulation are enhanced at strong horizontal buoyancy fronts, typical of the submesoscale, and, as demonstrated below, in some cases may dominate both wind and surface buoyancyflux-driven PV fluxes. These dynamics thus provide a direct link across spatial scales, whereby small-scale boundary layer turbulence, in the presence of submesoscale fronts, can alter the potential vorticity, and hence circulation, of the ocean gyres.

The broad significance of these processes is demonstrated in the context of the seasonal cycle of subtropical mode water formation in the North Atlantic. Ocean mode waters are defined by their anomalously low PV, and represent a key pathway for communicating a history of air-sea interaction into the ocean interior, exporting heat and carbon, and influencing the gyre-scale 
circulation (Hanawa and Talley 2001; Bates et al. 2002; Kwon and Riser 2004). The formation of ocean mode waters is fairly well explained by air-sea buoyancy fluxes; however, their seasonal destruction from late winter through the fall is less thoroughly understood. It has been hypothesized that mode water destruction involves turbulent mixing in the ocean interior (Qiu et al. 2006; Billheimer and Talley 2016), possibly influenced by double diffusion (Wong and Johnson 2003; Tsuchiya and Talley 1998). However, the more complex dynamics of PV relative to temperature, salinity, and velocity raises doubts that turbulent mixing unequivocally acts to destroy mode waters. In particular, turbulent diffusion of heat, salt, and momentum does not necessarily induce a downgradient flux of PV (Thorpe and Rotunno 1989; Keyser and Rotunno 1990) and therefore may not always mix away minima in PV associated with mode waters. Furthermore, following the impermeability theorem of Haynes and McIntyre (1987), interior mixing processes do not directly affect isopycnal PV budgets and hence cannot change the volume-integrated PV anomalies associated with mode waters. The impermeability theorem instead suggests that mode water destruction should involve processes that inject high PV into the mode water isopycnal layer at the ocean surface or bottom.

An important cause of surface PV flux is the air-sea flux of buoyancy (Nurser and Marshall 1991). However, analyses of the mode water seasonal cycle show that surface buoyancy fluxes lead to a net removal of PV, and hence the total mode water PV budget cannot be closed based on air-sea fluxes alone (Maze and Marshall 2011; Forget et al. 2011). A potential candidate for resolving this conundrum comes from considering recent observational and numerical modeling work, which suggests that the mode water formation regions are sites of active submesoscale turbulence (Shcherbina et al. 2013; Gula et al. 2014; Callies et al. 2015; McWilliams 2016). This implies that mode waters, rather than being formed in large, spatially homogeneous, outcropping regions, are the cumulative result of formation, destruction, and subduction over many small outcropping regions between submesoscale fronts, where frontal dynamics can modify the surface flux of PV (Thomas and Ferrari 2008). Here we demonstrate how the TTW circulation acts as a source of PV at submesoscale fronts, modifying the seasonal cycle of mode water formation and destruction, and reducing the total annual mode water PV removal by a factor of $\sim 3$, relative to the expectation based on surface buoyancy fluxes alone. Surface TTW PV fluxes in numerical models are also shown to be highly resolution dependent, with important implications for accurate large-scale modeling of the interior ocean circulation.
The manuscript is organized as follows. In section 2 we develop the theory of how the TTW circulation generates a surface PV flux. Simple scalings for the TTW PV flux are then derived and tested in idealized model runs of frontal spindown (section 3). In section 4 these ideas are applied to a realistic high-resolution model of the North Atlantic Subtropical Mode Water (Eighteen Degree Water), and it is demonstrated that these submesoscale processes are a leading-order term in the PV budget. In section 5 we discuss the relation of these nonconservative processes to adiabatic baroclinic mixed layer instabilities and boundary layer restratification, and major findings are summarized in section 6 .

\section{Theory}

\section{a. Potential vorticity}

The Ertel PV is given by

$$
q=\boldsymbol{\omega}_{a} \cdot \nabla b,
$$

where $\boldsymbol{\omega}_{a}=f \hat{\mathbf{k}}+\nabla \times \mathbf{u}$ is the absolute vorticity and $b=-g \rho / \rho_{o}$ is the buoyancy. It is possible to write a conservation equation for $q$ of the form (Marshall and Nurser 1992)

$$
\frac{\partial q}{\partial t}+\nabla \cdot \mathbf{J}=0
$$

where the "J vectors" are defined as

$$
\mathbf{J}=\underbrace{\mathbf{u} q}_{J_{A}} \underbrace{-\boldsymbol{\omega}_{a} D}_{J_{D}}+\underbrace{\nabla b \times \mathbf{F}}_{J_{F}} .
$$

The first term on the right-hand side of (3), $J_{A}$, gives the advection of PV. The second term gives the diabatic flux of PV, $J_{D}=-\omega_{a} D$, which arises from the turbulent buoyancy flux divergence in the buoyancy equation

$$
D=\frac{\partial b}{\partial t}+\mathbf{u} \cdot \nabla b
$$

The last term on the right-hand side of (3), $J_{F}=\nabla b \times \mathbf{F}$, arises from the turbulent momentum flux divergence in the horizontal momentum equations

$$
\mathbf{F}=\frac{\partial \mathbf{u}}{\partial t}+\mathbf{u} \cdot \nabla \mathbf{u}+\frac{1}{\rho} \nabla P+f \hat{\mathbf{k}} \times \mathbf{u} .
$$

The last two terms in (3) are therefore associated with nonconservative processes, as discussed in detail below.

Of particular utility is that when (2) is volume integrated between two isopycnal surfaces, it reduces to 


$$
\frac{\partial}{\partial t} \int q d V=-\left.\int J_{F}^{z}\right|_{z=0} d A-\left.\int J_{D}^{z}\right|_{z=0} d A,
$$

where $J_{F}^{z}$ and $J_{D}^{z}$ denote the vertical component of the frictional and diabatic terms from (3), respectively; and it is assumed for simplicity that the isopycnal outcrops at the surface but does not encounter topography. Physically, (6) states that the volume-integrated PV in an isopycnal layer changes only in response to the nonconservative fluxes integrated over the outcropping surface area (denoted by $A$ ). Interior nonconservative PV fluxes can be shown either to be oriented along buoyancy surfaces or to exactly balance the rate of movement of the buoyancy surfaces; hence, they do not contribute to the volume-integrated PV budget, a result known as the "impermeability theorem" (Haynes and McIntyre 1987; McIntyre and Norton 1990). Thus, for an outcropping mode water layer, the volume-integrated PV can be changed only by surface fluxes, a result that emphasizes that mixing processes outside the boundary layer cannot be invoked to close the volume-integrated mode water PV budget. Likewise, (6) continues to hold if the volume integral is taken over a fixed volume where fluxes of $q$ disappear at the horizontal and bottom boundaries, or in idealized periodic domains, as for instance in the idealized numerical model discussed in section 3 .

While the impermeability theorem highlights the unique role of boundary PV fluxes, an important point to emphasize is that turbulent processes in the boundary layer-but not necessarily directly at the surface-can generate a surface flux of PV, and hence through (6) modify the volume-integrated PV content of an isopycnal layer. To see this, consider a simplified example, where the horizontal buoyancy gradient and relative vorticity are assumed to be vertically uniform in the mixed layer. Assuming that turbulent mixing is dominated by the vertical component, the nonconservative terms can be written in the Reynolds-averaged framework as $\mathbf{F}=\partial / \partial z(\nu \partial \mathbf{u} / \partial z)$ and $D=\partial B / \partial z$, where $\nu$ is the combined turbulent and molecular viscosity, and $B$ represents the sum of both diffusive and nonlocal turbulent fluxes of buoyancy. The total vertical nonadvective PV flux can then be written as

$$
J_{F}(z)+J_{D}(z) \approx \frac{\partial}{\partial z}\left(-\omega_{a}^{z} B+f \nu \frac{\partial \mathbf{u}}{\partial z} \cdot \frac{\partial \mathbf{u}_{g}}{\partial z}\right)
$$

where $\mathbf{u}_{g}$ is the geostrophic component of the velocity, and for notational clarity we have dropped the superscript $z$ from this and all remaining $\mathrm{J}$ vectors discussed in this manuscript. This form highlights how the flux of PV depends on both the magnitude and the vertical structure of turbulent mixing, and we show below an example of how this can allow processes occurring within the boundary layer to modify the surface flux of PV.

In a well-mixed layer near the surface, which is expected to exist during times of surface buoyancy loss, the vertical component of the PV flux will also be approximately vertically uniform (as shown using scaling arguments in appendix A and from numerical results in section $3 b$ ). Thus, by integrating (7) to the depth where $B=0$, denoted by $h$ [which was termed the "convective" depth by Taylor and Ferrari (2010)], the vertically uniform PV flux near the surface can be written approximately as

$$
J(z) \approx \frac{f}{h}\left(B_{o}+\mathrm{EBF}+\mathrm{EBF}_{g}\right), \text { for } z>-h,
$$

where $B_{o}$ is the surface buoyancy flux defined as positive upward, $\mathrm{EBF}=(\rho f)^{-1}\left(\boldsymbol{\tau}^{w} \times \hat{\mathbf{k}}\right) \cdot \nabla_{h} b$ is the "Ekman buoyancy flux" (Thomas and Lee 2005), $\tau^{w}$ is the surface wind stress, and we have introduced

$$
\mathrm{EBF}_{g}=-\left.\nu \frac{\partial \mathbf{u}}{\partial z} \cdot \frac{\partial \mathbf{u}_{g}}{\partial z}\right|_{z=-h},
$$

the "geostrophic Ekman buoyancy flux" (cf. Bachman and Taylor 2016). Clearly the value of $\mathrm{EBF}_{g}$ will depend on $h$, which, based on both large-eddy and Reynoldsaveraged simulations, is expected at a front to often be less than the full turbulent boundary layer depth, denoted as $H$; that is, the turbulent mixing of momentum extends deeper than the convective depth (see, e.g., Taylor and Ferrari 2010; Thomas and Taylor 2010; section 3b). However, we also call attention to the fact that the current understanding of frontal effects on boundary layer turbulence is incomplete, and no general prognostic formulation currently exists for determining $h$ (cf. Taylor and Ferrari 2010). The formulation in (8) should therefore be understood as purely diagnostic, illustrating how both surface buoyancy forcing and the divergence of the turbulent mixing of momentum across the well-mixed surface layer generates a surface flux of PV.

Extensive prior work has confirmed that EBF can become a dominant term in both the surface buoyancy and PV budgets at the strong horizontal buoyancy gradients characteristic of the submesoscale (e.g., Thomas and Lee 2005; Thomas and Ferrari 2008; D'Asaro et al. 2011). These strong fronts are however also associated with large thermal wind shears, which implies $\mathrm{EBF}_{g}$ will also be enhanced through the mixing of geostrophic momentum, that is, $\mathrm{EBF}_{g} \approx-\left(\nu\left|\partial \mathbf{u}_{\mathrm{g}} / \partial z\right|^{2}\right)_{z=-h}$. Much of the remainder of this manuscript will therefore focus on quantifying the importance of $\mathrm{EBF}_{g}$ at the submesoscale, including determining a simple approximation that can be used to estimate the associated PV flux without requiring 
knowledge of the turbulent viscosity or convective depth (section 3), and providing an estimate of the contribution of $\mathrm{EBF}_{g}$ to the seasonal PV budget of the Eighteen Degree Water (section 4). However, before proceeding further, it is useful to first discuss how the physical origin of both $\mathrm{EBF}$ and $\mathrm{EBF}_{g}$ can be understood in terms of the cross-frontal advection of buoyancy, with important implications for how the turbulent mixing of momentum and buoyancy are coupled at a front.

\section{b. Interpretation in terms of frontal dynamics}

First, note that an alternate, physically intuitive expression for the total surface PV flux can be found using the formulation of Marshall et al. (2001) [their (13)], which can be rewritten as

$$
J=-\omega_{a}^{z}\left(\frac{\partial b}{\partial t}+\mathbf{u}_{i} \cdot \nabla b\right)
$$

where $\mathbf{u}_{i}$ is the inviscid component of the flow-that is, not associated with turbulent momentum mixing - that satisfies

$$
\mathbf{u}_{i}=\mathbf{u}-\frac{\mathbf{F} \times \hat{\mathbf{k}}}{\omega_{a}^{z}}
$$

The sign of the total PV flux will therefore be determined by whether the surface buoyancy is increasing or decreasing in time following the inviscid flow. Noting that the last term on the right-hand side of (11) is similar to an Ekman velocity $\mathbf{u}_{e}$ modified by the flow relative vorticity (cf. Wenegrat and Thomas 2017), the total surface PV flux [(10)] can be rewritten approximately as

$$
J \approx-f\left(D-\mathbf{u}_{e} \cdot \nabla b\right),
$$

in the limit of low Rossby number, highlighting the dependence of the PV flux on the turbulent mixing of buoyancy and the cross-frontal Ekman advection of buoyancy.

In this limit, the appropriate Ekman balance in the presence of a front, where the geostrophic shear is nonnegligible, is given by

$$
f \hat{\mathbf{k}} \times \mathbf{u}_{e}=\frac{\partial}{\partial z}\left[\nu\left(\frac{\partial \mathbf{u}_{e}}{\partial z}+\frac{\partial \mathbf{u}_{g}}{\partial z}\right)\right]
$$

with surface boundary condition (Thompson 2000; Thomas and Rhines 2002; Cronin and Kessler 2009)

$$
\rho \nu\left(\frac{\partial \mathbf{u}_{e}}{\partial z}+\frac{\partial \mathbf{u}_{g}}{\partial z}\right)=\boldsymbol{\tau}^{w}, \quad \text { at } \quad z=0
$$

Boundary layer turbulence thus couples the Ekman and thermal wind equations, such that ageostrophic flow will be generated at fronts by the turbulent mixing of geostrophic momentum, even in the absence of surface wind, as detailed in McWilliams et al. (2015) and Wenegrat and McPhaden (2016).

The balance (13) is linear, hence the total cross-frontal Ekman flow can be written as $\mathbf{u}_{e}=\mathbf{u}_{e}^{w}+\mathbf{u}_{e}^{\mathrm{TTW}}$, with separate contributions from the surface wind stress, satisfying a classic Ekman balance, and a component driven by the turbulent mixing of geostrophic momentum, which we refer to as the TTW flow. Then, using (3), (12), and (13), the vertical component of the surface PV flux resulting from the turbulent mixing of momentum can be approximated by

$$
J_{F} \approx \underbrace{f \mathbf{u}_{e}^{w} \cdot \nabla_{h} b}_{J_{F}^{\mathrm{WIND}}}+\underbrace{f \mathbf{u}_{e}^{\mathrm{TTW}} \cdot \nabla_{h} b}_{J_{F}^{\mathrm{TTW}}} .
$$

The relative strength of the TTW and wind-driven PV fluxes can be quantified as

$$
\gamma_{F}=\frac{u_{e}^{\mathrm{TTW}}}{u_{e}^{w}} \approx 0.1 \frac{H\left|\nabla_{h} b\right|}{f u^{*}},
$$

where $H$ is the turbulent boundary layer depth, $u^{*}=\sqrt{\tau^{w} / \rho}$ is the friction velocity, and we have approximated the viscosity as $\nu \approx 0.1 H u^{*}$ [for discussion and validation of this scaling, see McWilliams (2017)]. For conditions representative of the wintertime mode water formation regions $\gamma_{F} \sim 1$ (assuming $H \sim 300 \mathrm{~m}$, $\left|\nabla_{h} b\right| \sim(2 f)^{2} \mathrm{~s}^{-2}, u^{*} \sim 0.02 \mathrm{~m} \mathrm{~s}^{-1}$ ), suggesting the TTW flow will play a leading-order role in the dynamics.

A detailed discussion of wind-driven PV fluxes can be found in Thomas and Ferrari (2008). However, a point of particular relevance here is that the surface wind stress can alternately inject or remove PV, depending on the alignment of the wind stress with the horizontal buoyancy gradient. Thus, for a wind stress of large horizontal scale, when $J_{F}^{\text {WIND }}$ is integrated over a region of horizontally isotropic frontal features, it can be anticipated that alternate injection and removal of PV by the wind stress will largely cancel, as shown using high-resolution simulations of the North Atlantic Subtropical Mode Water formation region in section 4. In contrast, the TTW flow forms a closed secondary circulation in the across-front direction [as shown schematically in Fig. 1, and discussed in Wenegrat and McPhaden (2016) and McWilliams (2017)]. This circulation is thermally direct, with downgradient flow at the surface, which can be seen by noting that the TTW transport in the Ekman layer goes as $\mathbf{U}_{e}^{\mathrm{TTW}} \sim-\nu \nabla_{h} b /\left.\left(f^{2}\right)\right|_{z=-\delta_{e}}$, where $\delta_{e}=\sqrt{2 \nu / f}$ is the Ekman depth. Thus, unlike the wind-forced PV flux, the TTW component of the PV flux is always of the sense to inject PV at the surface, regardless of frontal orientation. 


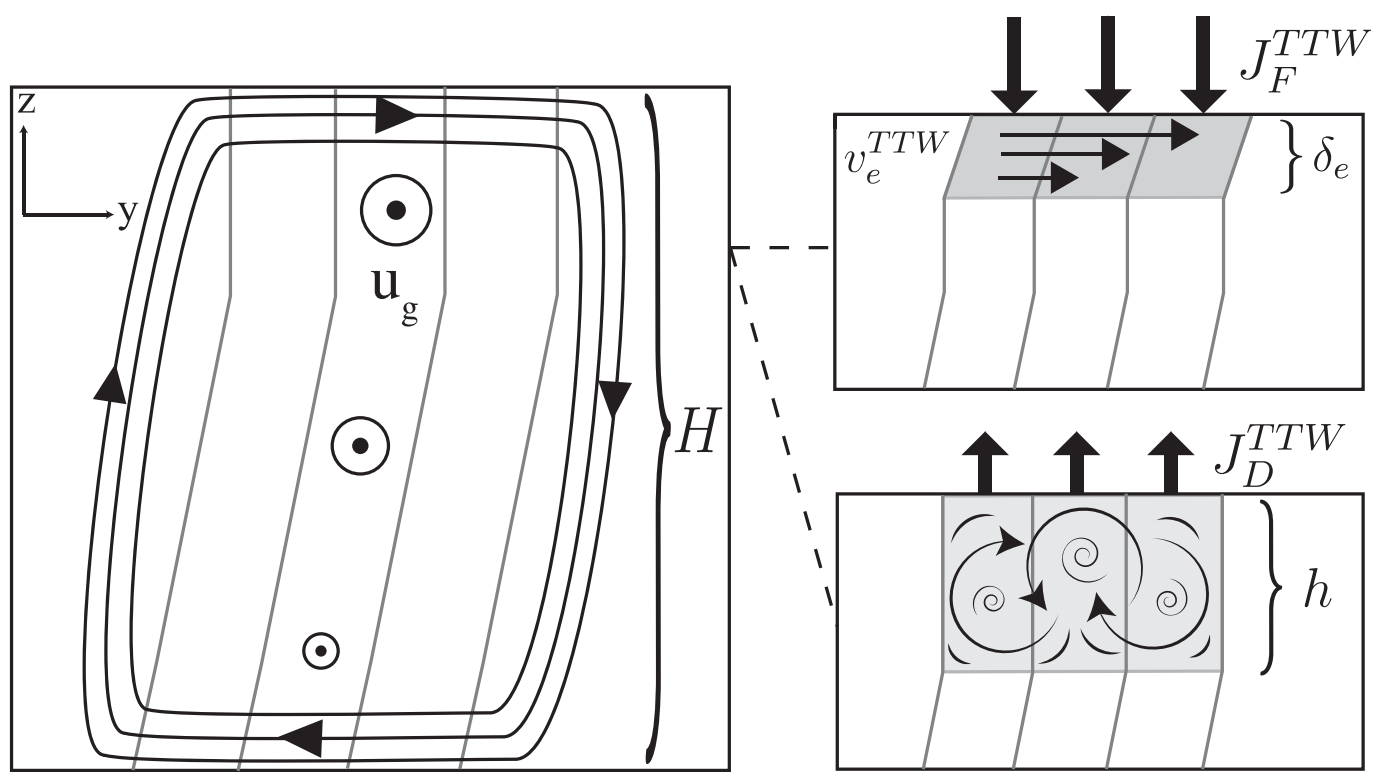

FIG. 1. Schematic of the TTW circulation and associated PV fluxes, as discussed in section 2. (left) The basic overturning circulation, where a horizontal buoyancy gradient (thin contours) generates a thermal wind shear, and in the turbulent boundary layer, a TTW overturning circulation in the across-front plane (Wenegrat and McPhaden 2016). (top right) The physical origin of the associated PV fluxes, where the sheared cross-frontal flow is restratifying, generates a positive buoyancy tendency at the surface (shown schematically by the shading). By (10), this indicates a downward flux of PV at the surface, $J_{F}^{\mathrm{TTW}}<0$ [see also (15)]. (bottom right) However, during conditions of surface buoyancy loss, or more generally when there exists a source of turbulence that maintains a well-mixed near-surface layer, the restratifying tendency of the TTW circulation will be opposed by enhanced turbulent mixing of buoyancy. Hence, for positive absolute vertical vorticity, the diabatic PV flux associated with the TTW circulation will be oriented upward, $J_{D}^{\mathrm{TTW}}>0$, indicating removal of PV at the surface. The total surface PV flux as a result of these processes is of the sense to increase the PV and is proportional to the geostrophic Ekman buoyancy flux distributed over the depth of the thermal boundary layer [see (8), (17), and the discussion in section 2].

The TTW circulation is also both restratifying and frontogenetic (Thompson 2000; McWilliams et al. 2015), as shown in Fig. 1. This leads to an evolution of the buoyancy field, which in turn affects the efficiency of both vertical and horizontal vertical mixing of buoyancy. This is a critical point, as, through (12), it can be seen that the total PV flux is determined by the difference between the cross-frontal Ekman advection and the turbulent mixing of buoyancy, which will itself be affected by the frontal dynamics and hence may not scale directly with the surface buoyancy flux. This issue is complicated by the fact that, generally, determining the balance between the rate of change, cross-frontal advection, and the turbulent of mixing of buoyancy is intractable to analytical methods, as it will involve both the nonlinear evolution of the buoyancy field caused by TTW advection and the effects of the changes in buoyancy on the resulting turbulence properties of the boundary layer. However, a simplified analytical analysis of this coupling is provided in appendix B, using an asymptotic expansion that assumes a thin Ekman layer relative to the full turbulent boundary layer depth, a limit that is consistent with the deep mixed layers found in the mode water formation regions.

The results of that analysis are summarized schematically in Fig. 1. As discussed above, the geostrophic shear in the turbulent boundary layer acts as a stress, driving a sheared flow down the buoyancy gradient that would tend to restratify the near-surface layer (Wenegrat and McPhaden 2016; McWilliams 2017). This restratification is however opposed by enhanced turbulent mixing of buoyancy, which at leading order keeps the Ekman layer unstratified and redistributes the advective buoyancy anomaly over a well-mixed near-surface layer of depth $h$. The net PV fluxes resulting from the TTW circulation are thus still of the sense to inject PV $\left(\partial b /\left.\partial t\right|_{z=0}>0\right)$, albeit at a rate reduced from that implied by the frictional component alone [(15)]. Thus, in the limit that $\delta_{e} / H \ll 1$, the total TTW PV flux, accounting for the coupled interaction between cross-frontal advection and turbulent mixing of buoyancy, can be written as

$$
J_{F}^{\mathrm{TTW}}+J_{D}^{\mathrm{TTW}} \approx f \frac{\mathrm{EBF}_{g}}{h} .
$$


Likewise, the same physical mechanisms giving rise to the partial diabatic compensation of $J_{F}^{\mathrm{TTW}}$ will also hold for the PV fluxes associated with $J_{F}^{\mathrm{WIND}}$. Thus, the total wind-driven PV flux, including these diabatic effects, should properly be approximated as

$$
J_{F}^{\mathrm{WIND}}+J_{D}^{\mathrm{WIND}} \approx f \frac{\mathrm{EBF}}{h},
$$

which is reduced by a factor of $\delta_{e} / h$ compared to the common approach of considering only the frictional component (i.e., $J_{F}^{\mathrm{WIND}}$ ) and ignoring the effect of EBF on the diabatic flux (cf. Thomas and Ferrari 2008).

The origin of the last two terms in (8) can thus be understood physically as resulting from a coupled interaction between the cross-frontal advection of buoyancy, by the combined wind-driven and TTW flow, and a partially compensating increase in the turbulent mixing of buoyancy, which appears implicitly in (8) through changes to the convective layer depth $h$. The term in parentheses in (8) thus acts as an effective buoyancy flux that determines the Lagrangian rate of change of the surface buoyancy following the inviscid flow, consistent with (10) (and Marshall et al. 2001).

\section{c. Scalings for the PV flux}

To develop a simplified scaling for the TTW component of the PV flux, which can be evaluated without knowledge of the viscosity and convective depth, we will consider the case of no wind stress and a surface buoyancy loss. The frictional TTW PV flux can then be approximated as

$$
J_{F}^{\mathrm{TTW}} \approx-c_{F} H\left|\nabla_{h} b\right|^{2},
$$

where the TTW velocity is scaled as $u_{e}^{\mathrm{TTW}} \sim \nu\left|\nabla_{h} b\right| /\left(f^{2} \delta_{e}\right)$, eddy viscosity as $\nu \sim H u^{*}$, the Ekman depth as $\delta_{e} \propto u^{*} / f$, and $c_{F}$ is a positive coefficient yet to be determined. ${ }^{1}$

The diabatic component can be approximated as

$$
J_{D} \approx \underbrace{f c_{s} \frac{B_{o}}{H}}_{J_{D}^{\text {BUOY }}}+\underbrace{c_{D} H\left|\nabla_{h} b\right|^{2}}_{J_{D}^{\text {TTW }}},
$$

which consists of a component resulting from surface buoyancy fluxes $J_{D}^{\mathrm{BUOY}}$ and a component resulting from the geostrophic Ekman buoyancy flux $J_{D}^{\mathrm{TTW}}$. Note that we have assumed that the turbulent vertical buoyancy flux divergence will remain similar, at least in some areaintegrated sense, to classic upright convection, such that

\footnotetext{
${ }^{1}$ In pure convective conditions, as considered in section 3 , the friction velocity $u^{*}$ is replaced by the convective velocity scale $w^{*}=\left(B_{o} H\right)^{1 / 3}$ (Large et al. 1994).
}

$D \approx\left(-B_{o}-\left.B\right|_{z=-H}\right) / H$. The entrainment flux at the base of the turbulent boundary layer is then absorbed into a constant coefficient, $c_{s}=1.2$, appropriate for conditions of surface buoyancy loss. ${ }^{2}$ The results of the following section are insensitive to this particular choice; however, it should be emphasized that it is not currently well understood how the classic one-dimensional conception of boundary layer turbulence is modified by the presence of a front (e.g., Taylor and Ferrari 2010; Bachman et al. 2017). We comment further on this in section 6.

The second term on the right-hand side reflects the physics discussed above (and in appendix B), with the expectation therefore that $c_{F} \geq c_{D}$. The use of constant coefficients can be seen, through (B28), to absorb a weak dependence on the Ekman number. A useful nondimensional parameter can then be formed to quantify the relative importance of $\mathrm{EBF}_{g}$ to the surface buoyancy flux in the total PV budget,

$$
\gamma_{q}=\frac{\left(c_{F}-c_{D}\right)}{c_{s}} \frac{H^{2}\left|\nabla_{h} b\right|^{2}}{\left|f B_{o}\right|} .
$$

For the model runs considered in section $3 \mathrm{c}$, we find $\left(c_{f}-c_{d}\right) / c_{s} \sim 0.04$. When $\gamma_{q} \gg 1$ the abovementioned scalings imply that the PV flux will be dominated by TTW fluxes, such that the upper-ocean gains PV regardless of the sign of the surface buoyancy flux. For $\gamma_{q} \ll 1$, PV fluxes will be dominated by surface buoyancy fluxes. We note also that $\gamma_{q}$ is proportional to the inverse of the $S$ parameter introduced in Mahadevan et al. (2012), although the physics captured here differ from the principally adiabatic restratification effects considered in that work (section 5).

\section{Idealized numerical experiments}

\section{a. Configuration of experiments}

To test these scalings, we run a suite of idealized numerical experiments using the MITgcm (Marshall et al. 1997). The model is run in hydrostatic mode, for a horizontally periodic domain extending $80 \mathrm{~km}$ in the alongfront $(x)$ direction, $100 \mathrm{~km}$ in the across-front $(y)$ direction, and $300 \mathrm{~m}$ in the vertical. The grid resolution is a uniform $500 \mathrm{~m}$ in the horizontal, with 50 stretched

\footnotetext{
${ }^{2}$ Parameterization of entrainment fluxes is itself the subject of a large body of literature (see, e.g., Kraus and Businger 1994; Deremble and Dewar 2012); however, the simple approach we take here is supported by both empirical and numerical results for pure convective conditions (Large et al. 1994; Taylor and Ferrari 2010), and further was found to give good agreement with numerical model results in testing both simplified 1D models, as well as the $3 \mathrm{D}$ models discussed in section 3 .
} 


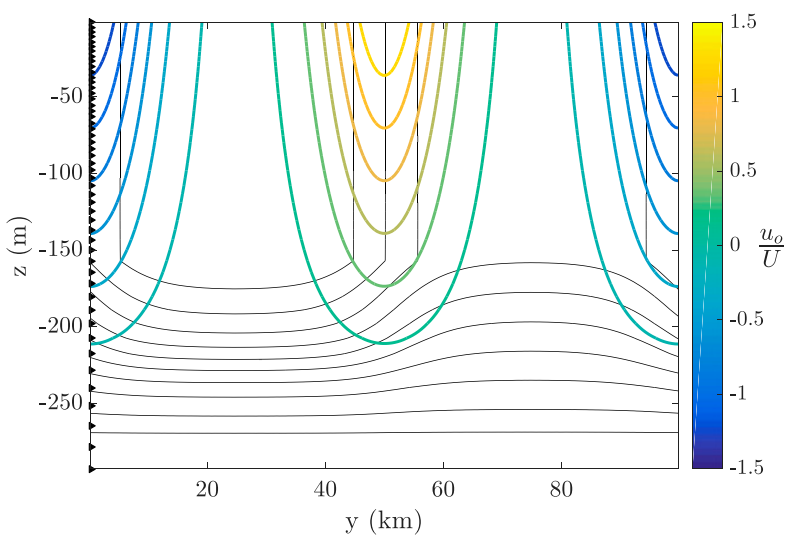

FIG. 2. Basic initial cross-frontal structure, with contours of buoyancy (black) and alongfront velocity (color). Velocity is normalized by $U=M_{o}^{2} H_{o} / f$. Black triangles along the $y$ axis denote the gridcell centers.

vertical levels, giving a vertical resolution that ranges from $\Delta z=3 \mathrm{~m}$ at the surface to $\Delta z=15 \mathrm{~m}$ at depth. For simplicity we utilize a linear equation of state dependent only on temperature; thus, $b=g \alpha T$, where $g$ is the gravitational acceleration, $\alpha$ is the thermal expansion coefficient, and $T$ is the temperature. Vertical mixing is parameterized using the $K$-profile parameterization (KPP; Large et al. 1994). This turbulence parameterization is chosen both for its physical basis in boundary layer similarity theory and for its common usage throughout ocean modeling.

The basic cross-frontal structure, and the initial geostrophically balanced velocity field, are shown in Fig. 2, and additional details of the model configuration are given in appendix C. This idealized model configuration is used to test the scalings developed in section 2 , by varying the initial horizontal buoyancy gradient $M_{o}^{2}$ and the strength of the applied surface heat flux $Q_{o}$ with parameters as given in Table 1 . To isolate the TTW effects, no surface wind stress is applied. Before discussing the results of the full parameter space, it is useful to first consider the evolution of a single model run.

\section{b. Example model run}

Snapshots of the temperature field for the model run with initial conditions $M_{o}^{2}=(2 f)^{2} \mathrm{~s}^{-2}$ and $Q_{o}=25 \mathrm{~W} \mathrm{~m}^{-2}$ provide a typical example of the frontal spindown process (Fig. 3). For these parameters $\gamma_{q} \sim 1$ such that both $\mathrm{EBF}_{g}$ and surface buoyancy fluxes are anticipated to be of similar importance. Early in the run, mixed layer instabilities (MLIs) develop with a horizontal wavenumber determined by the strength of the initial buoyancy gradient and the initial mixed layer depth (Boccaletti et al. 2007). By day 15, an inverse cascade of
TABLE 1. Parameters used for the idealized model discussed in section 3 .

\begin{tabular}{|c|c|c|}
\hline Symbol & Name & Values \\
\hline$M_{o}^{2}$ & $\begin{array}{l}\text { Initial maximum } \\
\text { horizontal buoyancy } \\
\text { gradient }\end{array}$ & $\left\{(1 f)^{2},(2 f)^{2},(4 f)^{2},(6 f)^{2}\right\} \mathrm{s}^{-2}$ \\
\hline$Q_{o}$ & Surface heat flux & $\{25,100,200\} \mathrm{W} \mathrm{m}^{-2}$ \\
\hline$\gamma_{q}$ & $\begin{array}{l}\text { Ratio of } J_{\text {TTW }} \text { and } J_{D}^{\text {BUOY }} \\
\text { from initial conditions } \\
{[(21)]}\end{array}$ & $10^{-2}-10^{2}$ \\
\hline$f$ & Coriolis frequency & $10^{-4} \mathrm{~s}^{-1}$ \\
\hline$H_{o}$ & Initial mixed layer depth & $150 \mathrm{~m}$ \\
\hline$N_{\text {INT }}^{2}$ & Interior stratification & $(64 f)^{2} \mathrm{~s}^{-2}$ \\
\hline$\Delta x, \Delta y$ & Horizontal grid spacing & $500 \mathrm{~m}$ \\
\hline$\Delta z$ & Vertical grid spacing & $3-15 \mathrm{~m}$ \\
\hline & Cross-frontal width & $10 \mathrm{~km}$ \\
\hline$\left(L_{x}, L_{y}\right)$ & Domain size & $(80 \mathrm{~km}, 100 \mathrm{~km})$ \\
\hline
\end{tabular}

energy leads to the development of frontal features with larger horizontal scale (Fox-Kemper and Ferrari 2008) and, importantly, strong frontogenesis occurs along the edge of the instabilities (Boccaletti et al. 2007). This frontogenesis leads to sharp horizontal buoyancy gradients, an example of which can be seen in Fig. 3, day 15, at the surface near $x=30 \mathrm{~km}, y=50 \mathrm{~km}$. These sharp frontal features rapidly enhance both the frictional and diabatic PV fluxes [(19) and (20), respectively] through their nonlinear dependence on the horizontal buoyancy gradient. Maximum domain-averaged horizontal buoyancy gradients occur shortly after day 20 (Fig. 4), associated with shallow boundary layer depths caused by frontal slumping. After day 25 the fronts are spinning down, exhausting the initial reservoir of available potential energy, decreasing the horizontal buoyancy gradients, and the boundary layer slowly deepens as a result of the surface buoyancy loss.

A time-integrated PV budget for this model run,

$$
\Delta q=-\int_{0}^{t} \int J_{F} d A d t-\int_{0}^{t} \int J_{D} d A d t
$$

is shown in Fig. 5, where $\Delta q=\int q(t) d V-\int q(0) d V$ is the cumulative change in PV integrated over the whole model volume. For the first 25 days of the model run, $\Delta q$ is a small residual term from the approximate cancellation between the frictional and diabatic fluxes. This is particularly notable in that the classical assumption, which ignores the frictional flux caused by TTW dynamics and assumes that the diabatic flux term is dominated by the surface buoyancy loss $J_{D}^{\text {BUOY }}$, would predict a steady removal of PV. However, here the sharp frontal features that develop (as evidenced in the relative vorticity field) inject PV, effectively offsetting the surface diabatic 


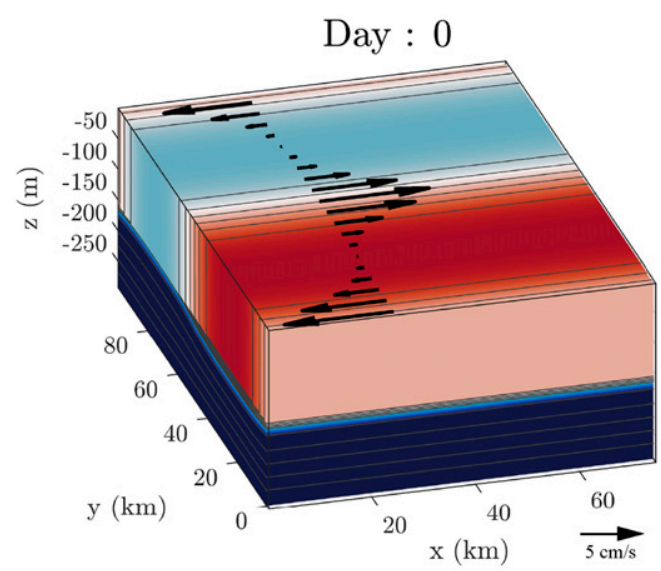

Day : 15

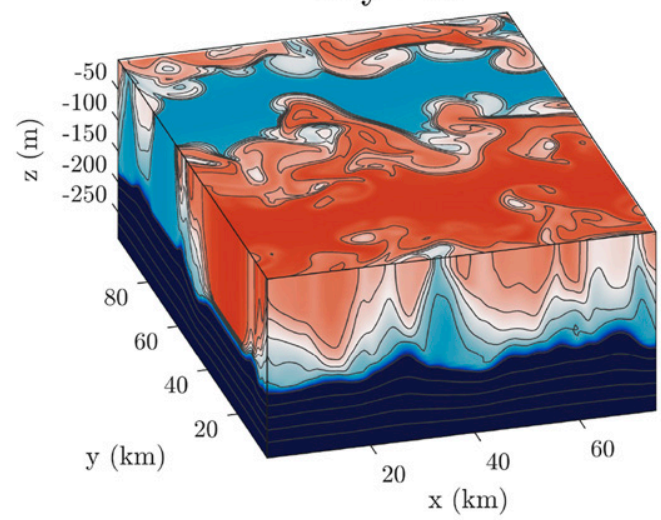

Day : 7.5

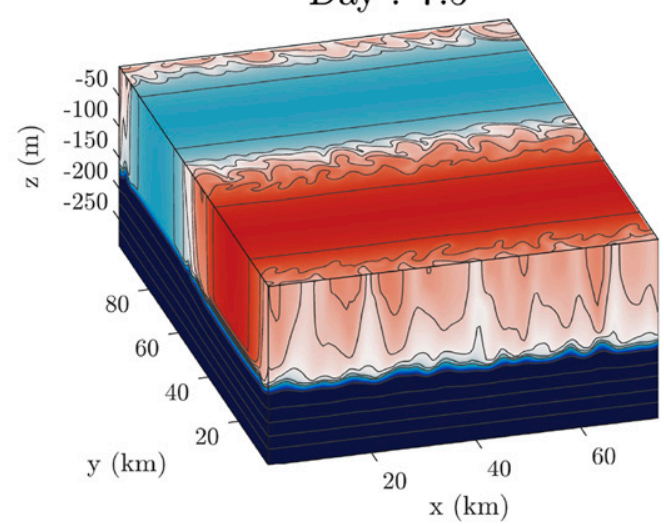

Day : 22.5

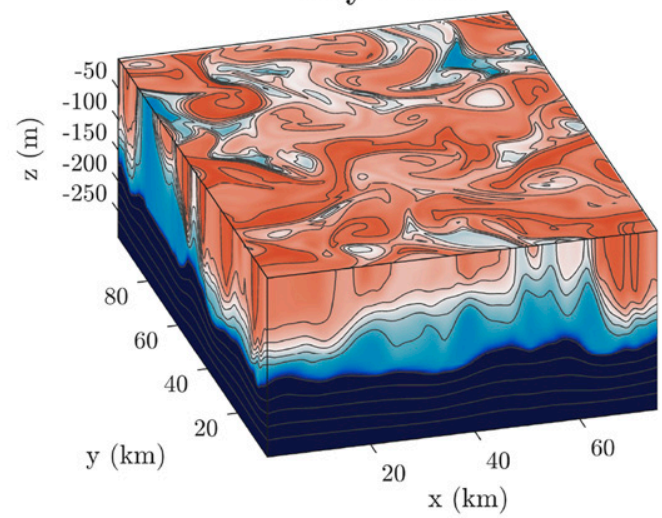

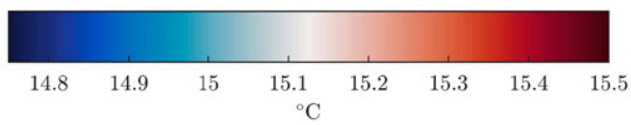

FIG. 3. Example evolution of the temperature field during frontal spindown for $M_{o}^{2}=(2 f)^{2} \mathrm{~s}^{-2}$ and $Q_{o}=25 \mathrm{~W} \mathrm{~m}^{-2}$ $\left(\gamma_{q} \sim 1\right)$. Gray contours of temperature are nonlinearly spaced so as to emphasize both deep and shallow variability. Vectors in top-left plot indicate the initial geostrophically balanced surface velocity.

removal of PV in the first half of the run. It is only later in the run, following day 25, when the fronts have begun to spin down (Fig. 4), that a cumulative PV loss develops as result of the constant surface buoyancy loss.

The strong frictional PV injection, and enhanced diabatic PV removal, associated with submesoscale fronts can be seen clearly in a snapshot from day 16 of the model run, shown in Fig. 6, with surface fluxes enhanced by two orders of magnitude over a scaling based on the surface buoyancy flux alone, $\left|f B_{o} / H_{o}\right|$. The total PV flux at the surface is spatially inhomogeneous, but because of the predominance of downward PV fluxes at locations of strong horizontal buoyancy gradients, the domain-averaged surface PV flux is oriented downward, such that the volume-integrated PV is increasing at this time. The reason for this can be seen in considering composite vertical flux profiles, conditionally averaged over regions of weak $\left[\left|\nabla_{h} b\right|<(6 f)^{2}\right]$ and strong $\left[\left|\nabla_{h} b\right|>(6 f)^{2}\right]$ fronts (Fig. 7).

Away from sharp fronts the flux terms in (7), $-B$ and $\nu \partial \mathbf{u} / \partial z \cdot \partial \mathbf{u}_{g} / \partial z$, form a combined profile that decreases approximately linearly from the surface buoyancy flux to zero at the base of the boundary layer (Fig. 7, top left). This gives a profile of vertical PV flux that is uniform, and positive, throughout much of the boundary layer, reflecting the removal of PV at the surface. In contrast, at the sharper fronts, both flux terms are enhanced by approximately an order of magnitude, with similar parabolic vertical structures of opposing sign (Fig. 7, top right). The enhancement of the turbulent momentum flux term is due to frontogenesis associated with mixed layer baroclinic instability, which increases the thermal 


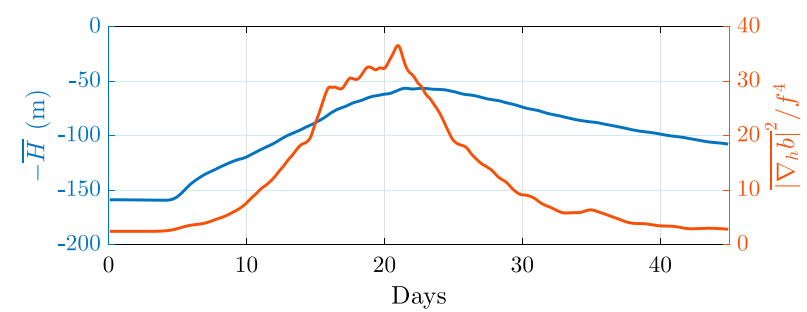

FIG. 4. Domain-averaged turbulent boundary layer depth $\bar{H}$ and normalized squared horizontal buoyancy gradient $\left|\nabla_{h} b\right|^{2} / f^{4}$, averaged over the boundary layer, for the model run shown in Fig. 3 and discussed in section $3 \mathrm{~b}$.

wind shear, modifying the turbulent flux both directly through changes in the geostrophic shear and through increased turbulent momentum fluxes resulting from the mixing of geostrophic momentum. The combined profile of $-B+\nu \partial \mathbf{u} / \partial z \cdot \partial \mathbf{u}_{g} / \partial z$ increases downward in the upper portion of the boundary layer, indicating a surface flux of PV into the boundary layer. The weak PV flux divergence over the upper half of the boundary layer (Fig. 7, bottom right) and convergence in the lower half of the boundary layer indicate how the destabilizing surface buoyancy loss acts to keep the upper portion of the boundary layer well mixed while PV accumulates in the lower portion of the boundary layer (consistent with the asymptotic analysis of appendix B).

As noted above, the cumulative effect of these submesoscale frontal processes on the PV budget will depend on the relative efficiency factors appropriate for scaling the frictional and diabatic removal, that is, $c_{F}$ and $c_{D}$ in (19) and (20), respectively. These are evaluated across all model runs in the following section.
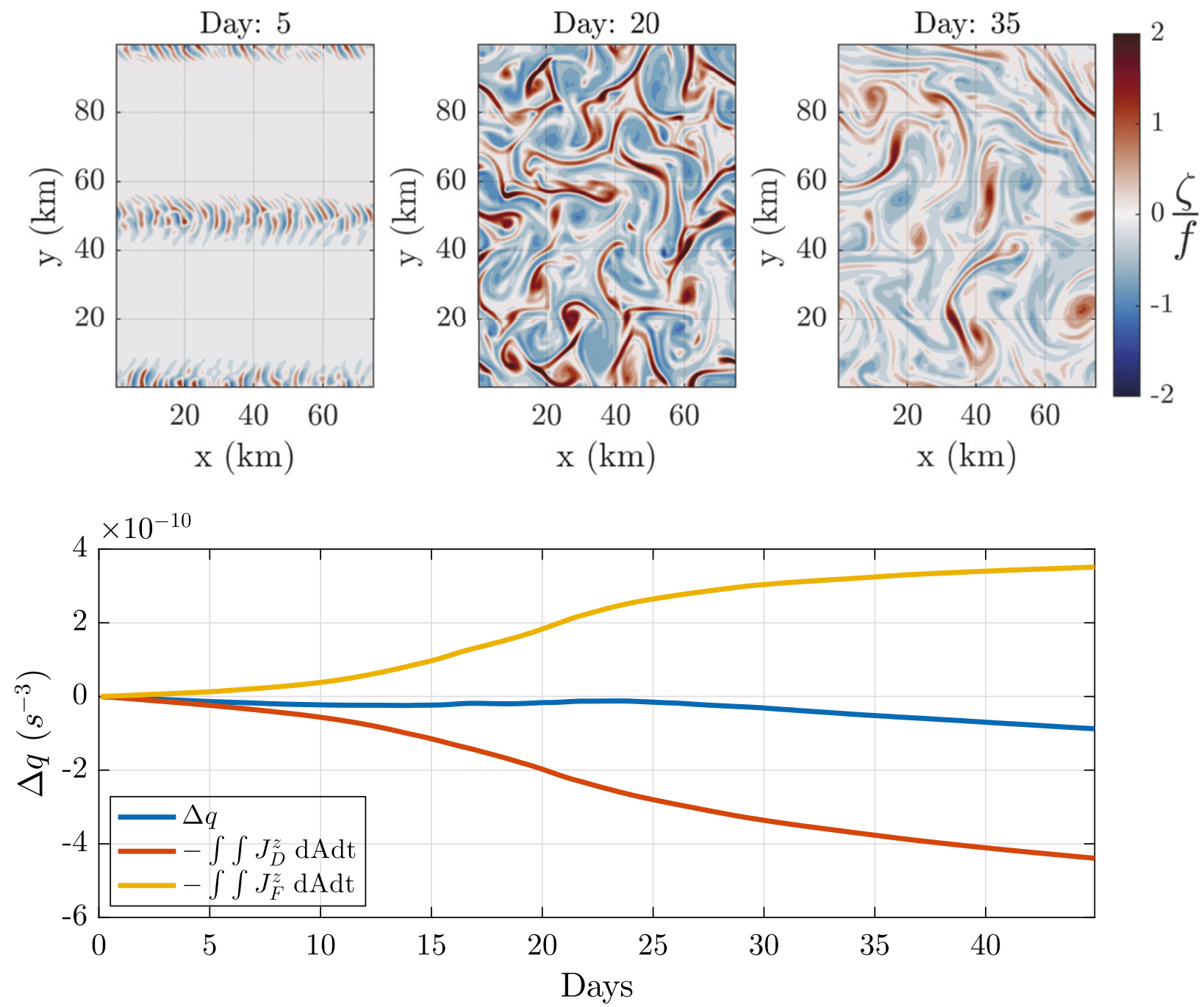

FIG. 5. (top) Surface vertical relative vorticity $\zeta=\hat{\mathbf{k}} \cdot \nabla \times \mathbf{u}$, normalized by $f$. (bottom) Cumulative PV budget. All plots are for the same simulation shown in Fig. 3. 

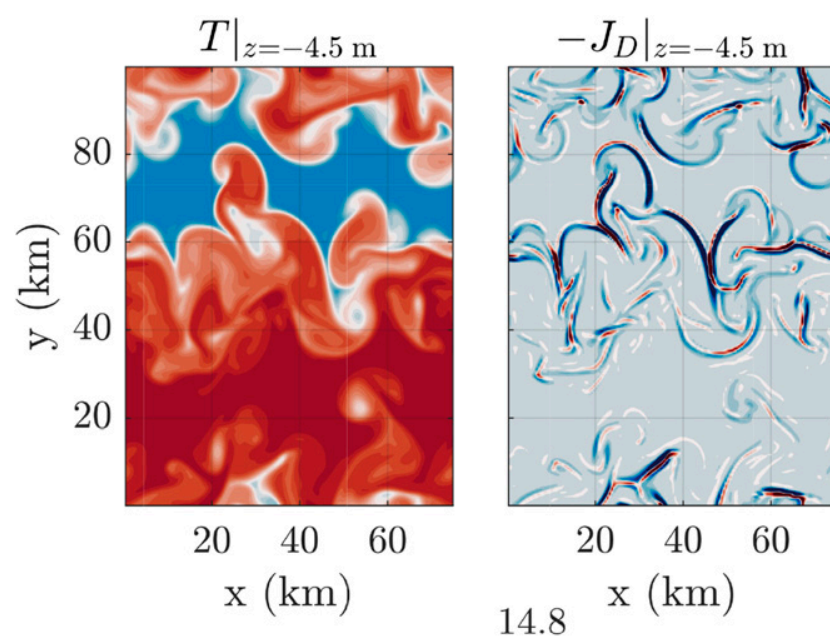
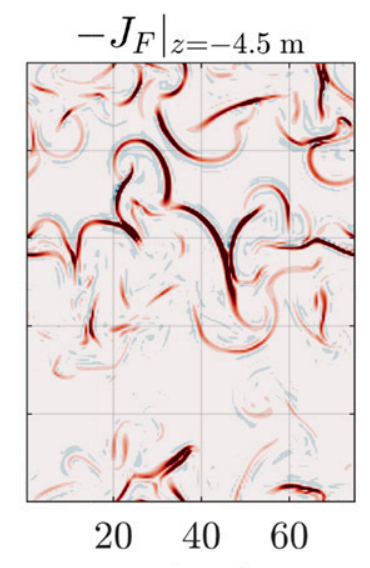

${ }^{\circ} \mathrm{C}$

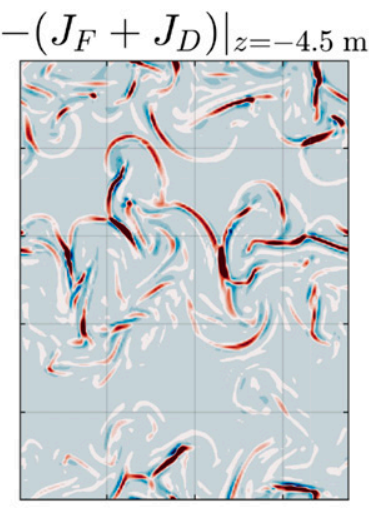

$20 \quad 40 \quad 60$ $\mathrm{x}(\mathrm{km})$

$-100$

0

100

\section{Normalized PV Flux}

FIG. 6. Snapshot of the near-surface temperature and vertical PV fluxes, from day 16 of the example model run discussed in section $3 \mathrm{~b}$ [with $M_{o}^{2}=(2 f)^{2} \mathrm{~s}^{-2}, Q_{o}=25 \mathrm{~W} \mathrm{~m}^{-2}, \gamma_{q} \sim 1$ ]. All J vectors are normalized by the surface buoyancy PV flux scaling $\left|f B_{o} / H_{o}\right|$.

\section{c. Comparison to theory}

Parameter space is explored using 12 model runs, formed as permutations of a set of four different values for the strength of the initial horizontal buoyancy gradient and a set of three values for the surface heat flux (Table 1). Each run is free to evolve from the initial conditions, and hence transient changes in the PV fluxes form an important part of the overall solutions; however, a useful indication of the relative importance of eddy versus surface effects for a model run is given by $\gamma_{q}[(21)$; Table 1] evaluated using the initial conditions, values of which span four orders of magnitude.

Estimates of (19) and (20) are calculated at each grid point for every output time step, and then averaged in space. The values of the efficiency factors are then found through linear regression with the $\mathrm{J}$ vectors calculated directly from the model output (appendix C). For $J_{D}$ the regression is performed between the scaling estimate and $J_{D}^{\mathrm{NUM}}-J_{D}^{\mathrm{BUOY}}$, that is, the difference between the model diabatic PV flux and the estimate based on the surface boyancy flux scaling alone. As shown in Fig. 8, the simplified scalings very accurately reproduce the model PV fluxes across parameter space, with best-fit efficiency factors of $c_{F}=0.2$, and $c_{D}=0.15$. The total PV flux as a result of the geostrophic Ekman buoyancy flux is therefore approximately

$$
J_{\mathrm{TTW}}=J_{F}^{\mathrm{TTW}}+J_{D}^{\mathrm{TTW}} \approx-0.05 H\left|\nabla_{h} b\right|^{2} .
$$

The success of the constant coefficient scalings in fitting the model results across all of parameter space implies that the results of appendix B, which were derived under an assumption of a thin Ekman layer, holds even for cases where $\delta_{e} / H$ is not necessarily small. Additional model runs were also performed using $H_{o}=25 \mathrm{~m}$, where $\delta_{e} / H$ would presumably be larger, and were found to give similar results (not shown). Likewise, the dependence of the total PV flux on the time-varying ratio $\delta_{e} / h$, indicated in the analysis of appendix B, appears to be of secondary importance in the domain-integrated PV budgets. This is not to say that this ratio remains constant, and inspection of the evolution of individual model runs suggests that this ratio varies spatially and temporally, reflecting the evolution of the frontal spindown process. Rather, in the volume-integrated PV budget, constant coefficients suffice to explain the area-integrated surface PV fluxes. Some caution is thus warranted in extrapolating these coefficients to a single submesoscale front or in conditions that differ greatly from those considered here, although these results, and the analysis of section 2 and appendix B, suggests a general expectation that the TTW circulation is a source of $\mathrm{PV}$, that is, $c_{F} \geq c_{D}$.

\section{d. Isopycnal PV budget}

Before considering a realistic model of the North Atlantic in section 4, it is useful to consider an isopycnal layer PV budget in the idealized domain to illustrate 

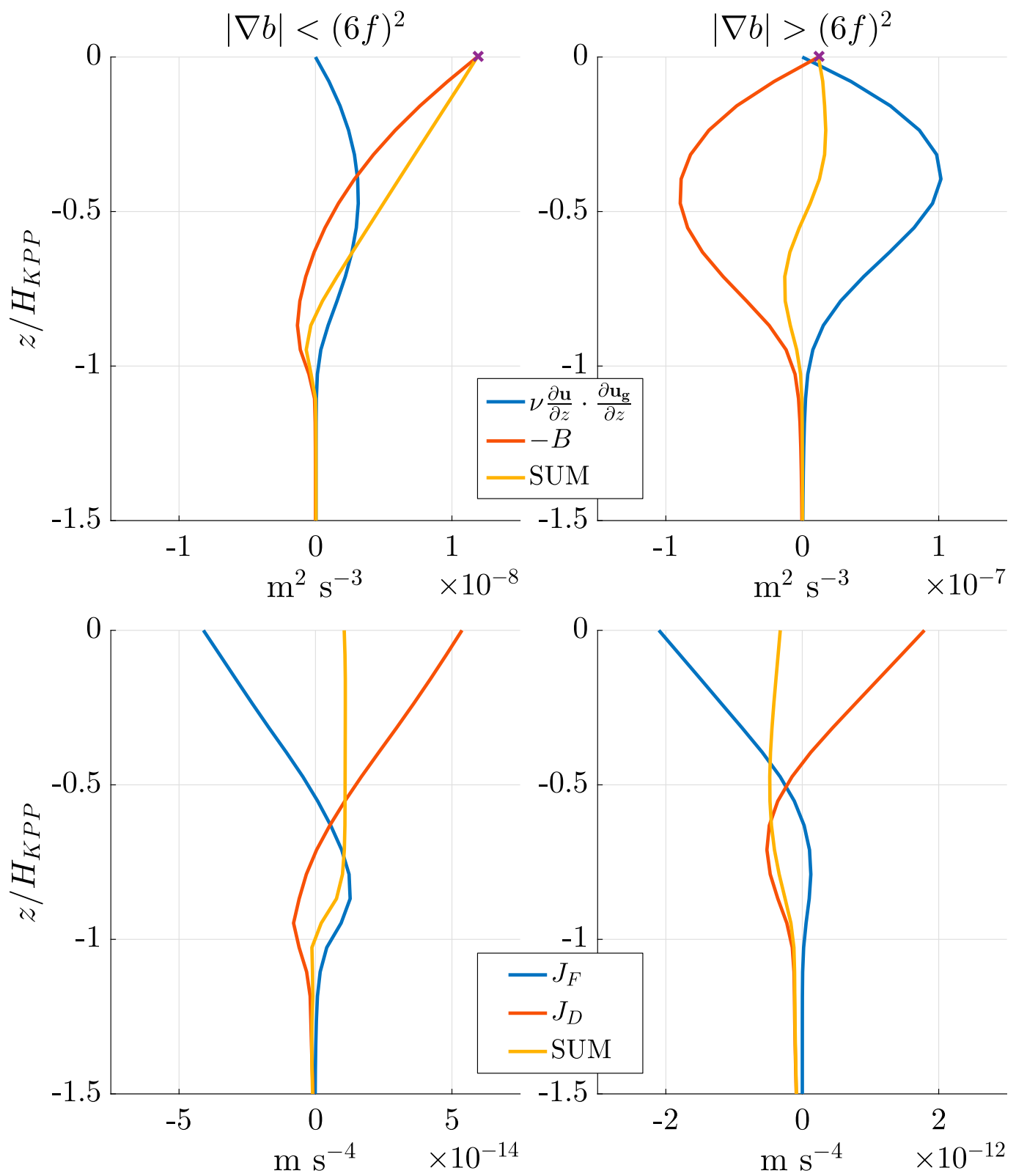

FIG. 7. Composite vertical profiles, conditionally averaged over regions of (left) weak horizontal buoyancy gradients and (right) strong horizontal buoyancy gradients, over \pm 1 inertial period centered at day 16 of the simulation discussed in section $3 \mathrm{~b}$, as shown in Fig. 6. To form these composites, individual profiles are first interpolated to a vertical coordinate that is normalized by the local (in space and time) KPP boundary layer depth and then averaged using the $5 \%$ trimmed mean to exclude outliers. In the top row, the value of the surface buoyancy flux $B_{o}$ is indicated by the $\times$ symbol.

how these processes may affect the mode water PV budget during the seasonal transition from winter to spring. To do this we run the same idealized model configuration [with $M_{o}^{2}=(4 f)^{2} \mathrm{~s}^{-2}$ ] with a surface buoyancy loss that decreases linearly with time (Fig. 9), mimicking the winter to spring transition in surface heat flux. A PV budget is taken over an isopycnal layer, which for illustration of the mode water problem can be considered as spanning $17^{\circ}-19^{\circ} \mathrm{C}$. At the beginning of the run, the isopycnal layer outcrops on the northern side of the domain (Fig. 9); later, despite the surface buoyancy loss, MLIs restratify the surface layer. By day 15 the isopycnal is almost completely capped, isolating it from further PV changes resulting from surface fluxes. 

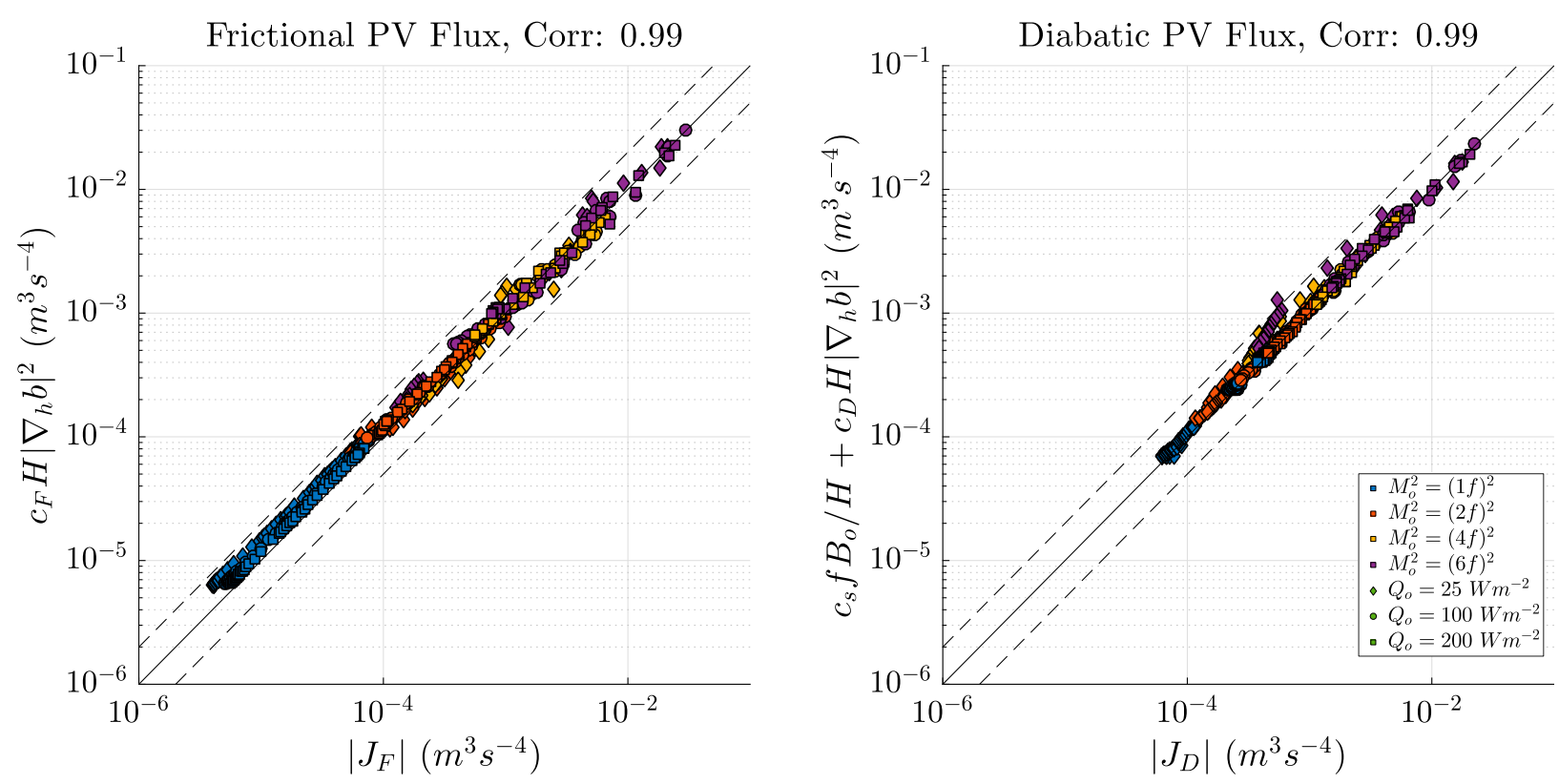

FIG. 8. Comparison of theoretical scalings ( $y$ axes) and model output ( $x$ axes) for the 12 model runs discussed in section 3c (Table 1). Daily averaged values are plotted for visual clarity, with initial conditions for each run indicated by a combination of marker color and shape (legend). The correlation coefficients for the 2 -h output fields are given above each plot. The $1-1$ line is indicated by a solid black line in each plot, and the dashed black lines indicate agreement to within a factor of 2.

Considering the cumulative PV budget for this isopycnal layer, it can be seen that between days 7 and 15 there is a rapid frictional injection of PV into the layer, leading to $\Delta q>0$. The significance of this can be seen in comparison with the PV loss as a result of the surface buoyancy flux alone (Fig. 9, dashed line), which is of the opposite sign and smaller in magnitude. This idealized configuration thus shows that outcropping fronts can become sufficiently sharp, sufficiently early in the spindown process, to allow isopycnal layers to accumulate a significant gain of $\mathrm{PV}$ before the layer is subducted as a result of the eddydriven restratification. Understanding these effects in the context of a realistic ocean model of the North Atlantic mode water formation region is the focus of the following section.

\section{Realistic model of the North Atlantic Subtropical Mode Water}

In this section we evaluate the impact of submesoscale processes in the seasonal PV budget of the North Atlantic Subtropical Mode Water, or Eighteen Degree Water (EDW), using a realistic, submesoscale permitting, model of the Gulf Stream region. The model is implemented using the Regional Ocean Modeling System (ROMS; Shchepetkin and McWilliams 2005), with configuration details described at length in earlier works such as Gula et al. (2014, 2015). We consider a large region of the northwest Atlantic, run at $1.5-\mathrm{km}$ horizontal resolution, with boundary conditions provided by nesting from a larger domain run at $\sim 5-\mathrm{km}$ resolution (Fig. 10). Surface forcing is provided by climatological heat flux, evaporation and precipitation, and surface wind stress constructed from climatology plus daily winds with variance close to climatological values (see Gula et al. 2015). The use of climatological forcing excludes the possible importance of air-sea feedbacks, although a correction term of $30 \mathrm{~W} \mathrm{~m}^{-2}{ }^{\circ} \mathrm{C}^{-1}$ is applied to the surface heat flux based on the difference between the modeled SST and the climatological SST. The model is run for 16 months, and we analyze the mode water formation season over the last 12 months. Given the single realization of the annual cycle, and the other limitations mentioned above, the results of this section are best treated as providing an extension of the findings of section 3 to a model with more complete and realistic physics, rather than a definitive exploration of the importance of these processes for the EDW water in particular (see, e.g., Maze et al. 2013).

A section through the domain is shown in Fig. 11, showing the thick EDW layer south of the Gulf Stream front. The mode water appears as a distinct peak in the density distribution, centered at $1026.1 \mathrm{~kg} \mathrm{~m}^{-3}$, slightly lighter than observed values near $1026.4 \mathrm{~kg} \mathrm{~m}^{-3}$ (McCartney 1982), and hence for this analysis we 

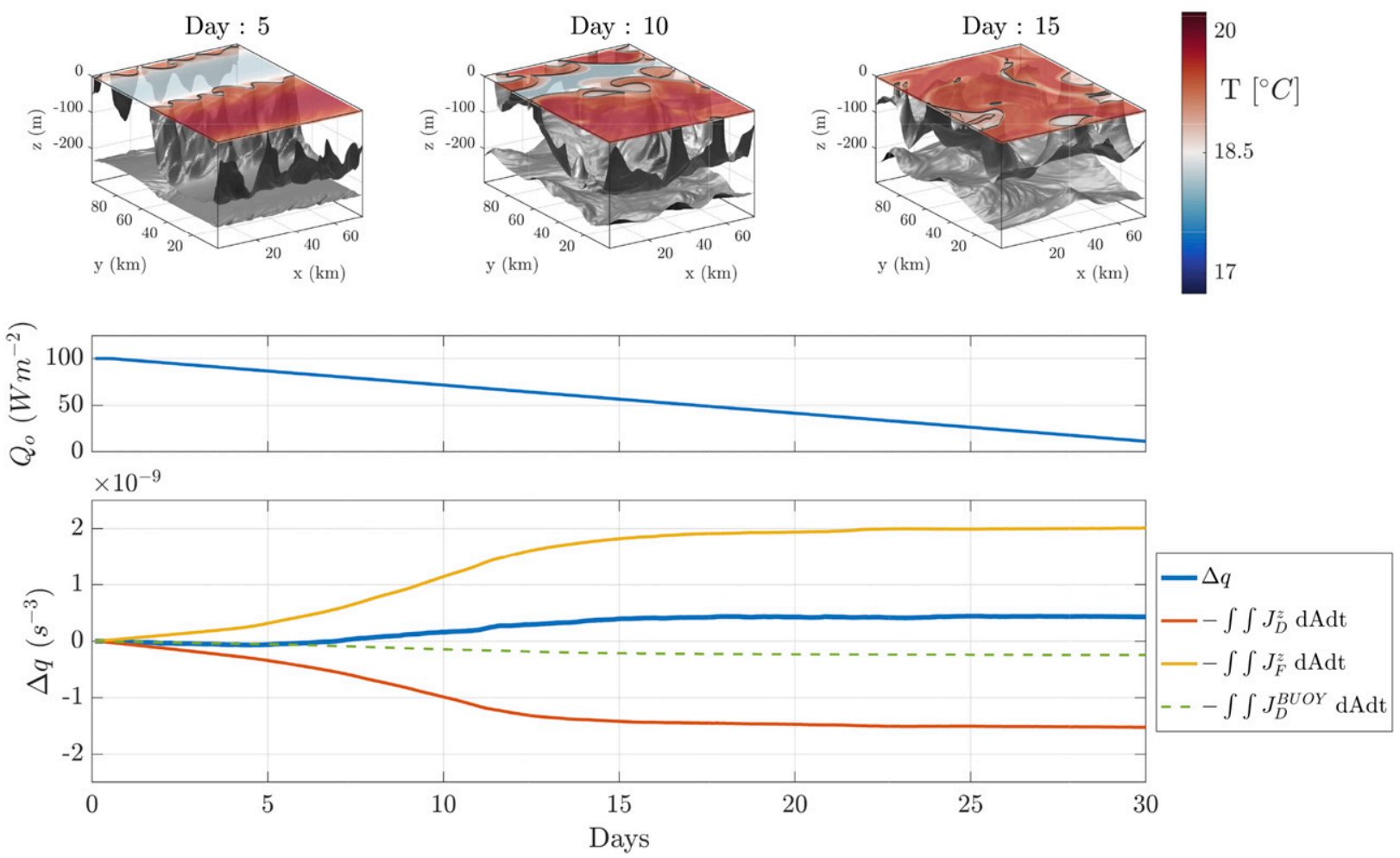

FIG. 9. Example isopycnal layer PV budget as discussed in section $3 \mathrm{~d}$. (top) Bounding isosurfaces $\left(T=17^{\circ} \mathrm{C}\right.$ and $T=19^{\circ} \mathrm{C}$, in gray) and the surface temperature field (color scale). (middle) Time-varying surface heat flux. (bottom) Cumulative change in the isopycnal-layer $\mathrm{PV}$ with components indicated in the legend.

define the mode water layer using a density criteria of $\rho=$ $1026.1 \pm 0.2 \mathrm{~kg} \mathrm{~m}^{-3}$. The contribution of surface buoyancy fluxes, wind-driven fluxes, and the TTW flux [(23)] to the annual mode water budget are evaluated from scalings and shown in Fig. 12. These contributions are calculated following $J(x, y)=1 / T \int_{0}^{T} J(x, y, t) G(x, y, t) d t$, where $T=$ $1 \mathrm{yr}$ and

$G(x, y, t)= \begin{cases}1, & 1025.9 \mathrm{~kg} \mathrm{~m}^{-3} \leq\left.\rho\right|_{z=0} \leq 1026.3 \mathrm{~kg} \mathrm{~m}^{-3} \\ 0, & \text { otherwise }\end{cases}$

Surface buoyancy fluxes generally remove PV, except for a region in the northern Gulf Stream, associated with surface warming of subpolar gyre water. The net flux of PV as a result of $J_{\mathrm{TTW}}$, evaluated using the approximation (23), is of comparable magnitude to the surface buoyancy PV fluxes, suggesting the importance of these processes in the annual mode water PV budget. The wind-driven flux of PV is strongest along the Gulf Stream front but is largely compensated between injection and removal, consistent with the analysis of a more coarsely resolved ocean state estimate by Maze and Marshall (2011).
The seasonal budget of the mode water volumeintegrated PV is calculated over a subdomain (indicated in top-left panel of Fig. 12), encompassing the regions of the largest surface fluxes, shown in Fig. 13. The mode water outcrops in this domain in early November, with PV being removed until March, followed by a brief period of PV injection until the mode water is subducted in May. The scalings developed in section 3 follow the modeled changes in PV closely, indicating that the reduction of PV loss in the model can be attributed to PV injection by TTW circulations. Notably, the rate of wintertime PV removal is reduced by approximately $50 \%$, and the formation season shortened by approximately 3 weeks, relative to the expectation from surface fluxes alone. The cumulative PV budget (Fig. 13, bottom) indicates that there is a net loss of mode water PV in this subdomain, although the total PV loss is only approximately $30 \%$ of the loss that would be expected based on surface buoyancy fluxes alone. These findings are consistent with the results of Lévy et al. (2010), who observed an increase in stratification of the mode water in an idealized gyre when the submesoscale was resolved. Likewise, while volumeintegrated PV does not uniquely determine the mode 


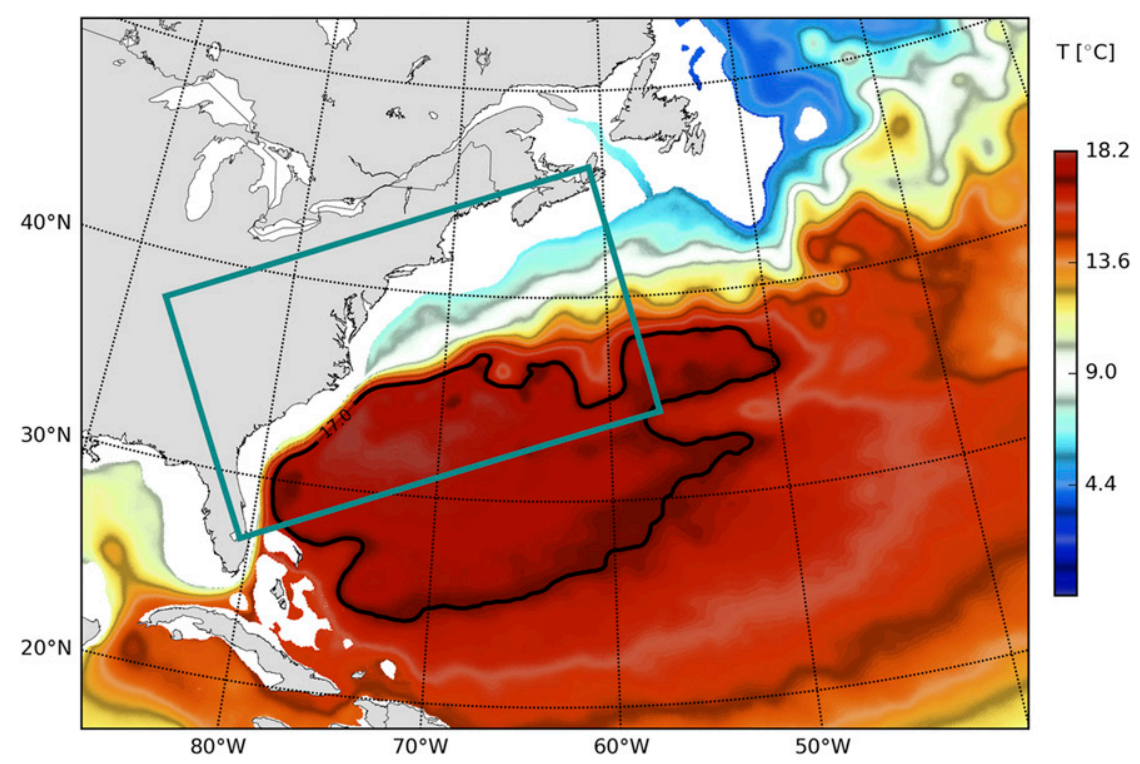

FIG. 10. Temperature at 400-m depth in the parent simulation of the North Atlantic (color scale). The domain of the $1.5-\mathrm{km}$ run is indicated in green. The heavy black line indicates the $17^{\circ} \mathrm{C}$ contour, often treated as the lower boundary of the EDW.

water volume (Deremble et al. 2014), the reduction of mode water PV loss relative to surface fluxes echoes observational analyses that find the volume of subducted mode water is greatly reduced from that formed by surface fluxes (Kelly and Dong 2013).

The volume-integrated PV varies by a factor of 2 over the seasonal cycle, with a seasonal minimum in late February. After this seasonal minimum, advective fluxes through the edges of the subdomain replenish the volume-integrated mode water PV, and the final cumulative loss of PV through the surface is balanced entirely by the cumulative advective fluxes. This is consistent with the analysis of Deremble and Dewar (2013); however, here the cumulative nonconservative PV loss is $30 \%$ of the average mode water PV in the model domain, indicating a renewal time scale of only $\sim 3$ years, as opposed to the $\sim 100$-yr time scale they find. These differences may be partly due to Deremble and Dewar (2013) considering annually averaged PV, excluding the seasonal cycle of mode water PV creation and destruction, over a control volume that encompassed less of the formation region than the domain considered here (Fig. 10). A full comparison between our results and prior work on the relationship between mode water PV and volume would be confounded by differences in model resolution, our single realization of an annual cycle, and our model domain covering an incomplete portion of the total mode water volume. However, the results of our analysis suggest that surface PV fluxes may induce large seasonal variability of the mode water PV and lead to a cumulative annual PV flux that represents a significant portion of the total mode water PV. Submesoscale processes greatly modify these surface PV fluxes and hence may exert a strong control on the gyre-scale circulation through their effects on the mode water PV budget.

One of the implications of the above analysis, and (23), is that the potential vorticity budget of the mode water in numerical models will be strongly dependent on the strength of the resolved surface buoyancy fronts. An example of the effect of decreased resolution can be found by averaging the model fields to a $10.5-\mathrm{km}$ grid, equivalent to the resolution of what would currently be considered a very high-resolution global ocean model, and then recalculating the PV

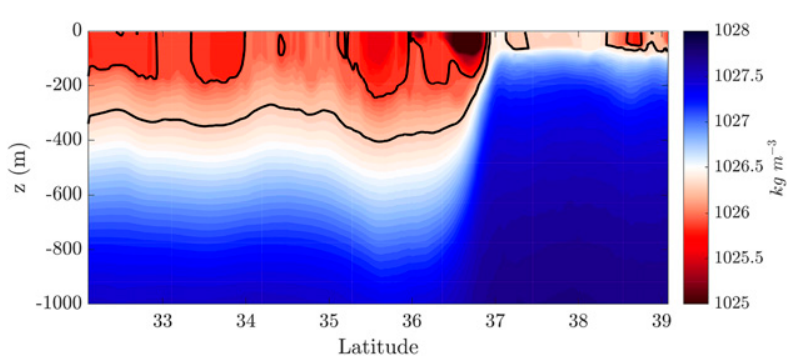

FIG. 11. Snapshot of the wintertime density field in the $1.5-\mathrm{km}$ model run along the section indicated in Fig. 12. The isopycnal layer used here to define the mode water is outlined with a heavy black line. 

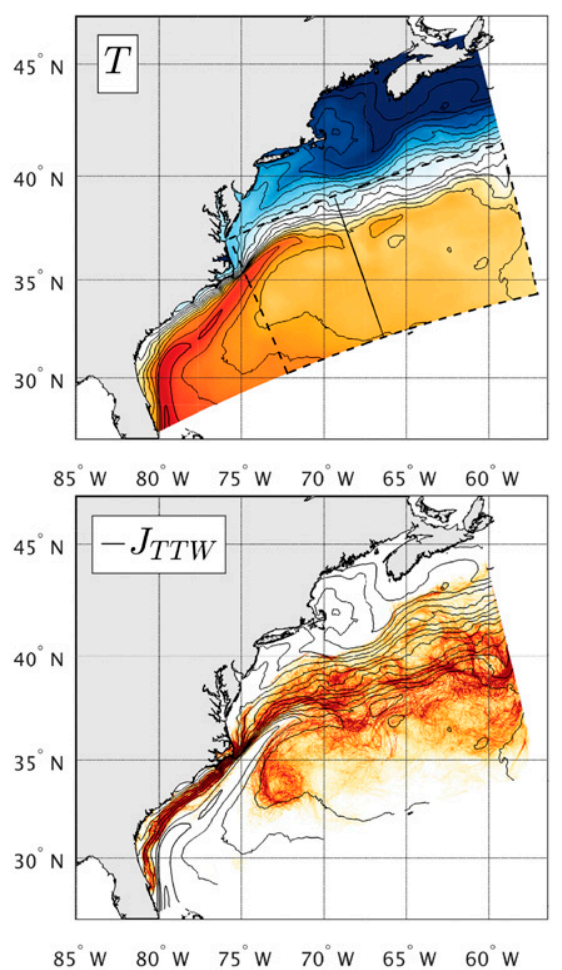

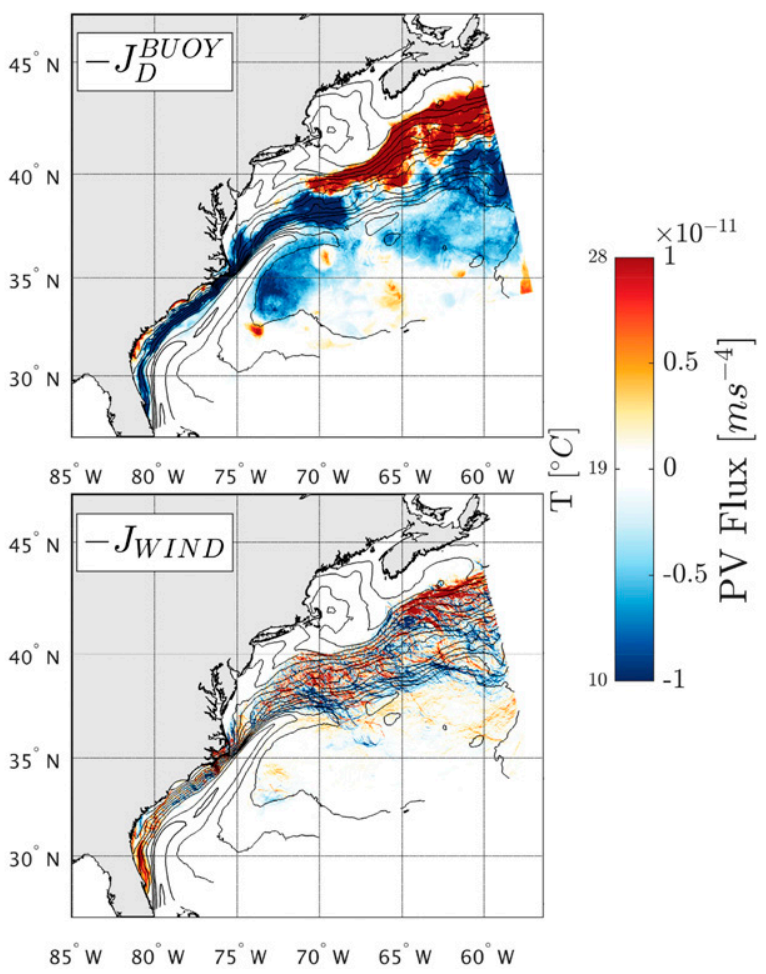

$85^{\circ} \mathrm{W} \quad 80^{\circ} \mathrm{W} \quad 75^{\circ} \mathrm{W} \quad 70^{\circ} \mathrm{W} \quad 65^{\circ} \mathrm{W} \quad 60^{\circ} \mathrm{W}$

FIG. 12. Annual averaged surface fields from the $1.5 \mathrm{-km}$ run. The $\mathrm{J}$ vectors are evaluated from the scalings given in the text and are multiplied by -1 to indicate the sign of their tendency to change PV. Annual averages are calculated over the time-varying mode water outcropping, as discussed in section 4. (top left) Surface temperature, (bottom left) $J_{\mathrm{TTW}}$ is defined in (23), (bottom right) $J_{\mathrm{WIND}}$ is approximated as $J_{\mathrm{WIND}} \approx f \mathrm{EBF} / H$, and (top right) $J_{D}^{\mathrm{BUOY}}$ is defined in (20). In each plot the thin contours indicate the annual average surface temperature field. In the top-left plot, the thin solid line indicates the section used in Fig. 11 and the dashed box indicates the domain used for Fig. 13.

fluxes from the scalings. Figure 14 shows $J_{D}^{\mathrm{BUOY}}$ and $J_{\text {TTW }}$ calculated using $1.5-$ and $10.5-\mathrm{km}$ fields. The surface PV flux $J_{D}^{\mathrm{BUOY}}$ is essentially unchanged between the two resolutions, and the area-integrated PV flux in the low-resolution (LR) case is essentially identical to the high-resolution (HR) model, reflecting the large scales of the atmospheric forcing. A caveat to this result is that averaging the high-resolution run does not capture the effect of resolution on the mean mixed layer depth, a mechanism that would be expected to somewhat reduce $J_{D}^{\mathrm{BUOY}}$ at low resolution. The PV injection as a result of TTW dynamics is, however, strongly dependent on resolution, and only $2 \%$ of the TTW PV flux is captured at $10.5 \mathrm{~km}$. This suggests that numerical models that do not resolve these submesoscale processes may accurately reproduce the surface removal of PV caused by air-sea fluxes but will miss an important submesoscale source of PV, with direct implications for the PV distribution in the gyre interiors, and hence the resulting gyre circulations. Likewise, we note that the $1.5-\mathrm{km}$-resolution domain used here is most properly considered only as submesoscale permitting and thus likely provides only a lower bound on the importance of submesoscale PV fluxes.

\section{Turbulent thermal wind restratification}

One of the principal topics driving interest in the ocean submesoscale is the tendency of submesoscale processes to restratify the near surface, and it is now widely recognized that submesoscale restratification is likely important to a wide range of physical, and biogeochemical, processes. As such, important steps have been taken toward parameterizing the effects of these processes within models that do not explicitly resolve the relevant length scales (Fox-Kemper and Ferrari 2008; Fox-Kemper et al. 2008, 2011; Bachman et al. 2017). The theory developed in section 2 , and tested in section 3 , suggests that, beyond submesoscale instabilities, turbulent nonconservative processes at the submesoscale likely also modify the boundary layer in critical ways. This is further supported by application of these ideas to a high-resolution 

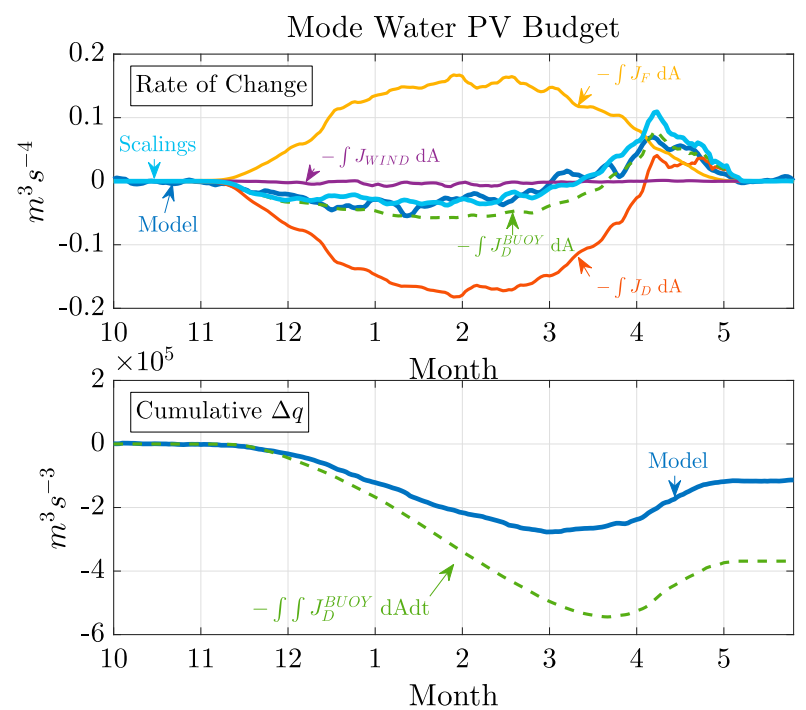

FIG. 13. Seasonal PV budget for the subdomain indicated in Fig. 12. (top) The rate of change, with Model $\equiv \partial / \partial t \int q d V+\int \mathbf{u} q \cdot \mathbf{n} d \Sigma$, i.e., the rate of change of $\mathrm{PV}$ in the isopycnal layer after accounting for advective fluxes through the edges of the subdomain (denoted by $\Sigma$ ). The total approximation to the right-hand side of the PV equation is given by Scalings $\equiv-\int\left(J_{D}^{\mathrm{BUOY}}+J_{\mathrm{TTW}}+J_{\mathrm{WIND}}\right) d A$, defined as in Fig. 12. (bottom) The time-integrated change in PV (Model $\equiv \Delta q+\iint \mathbf{u} q \cdot \mathbf{n} d \Sigma d t$ ). submesoscale-resolving simulation of the mode water formation region in the North Atlantic (section 4).

Notably, (10) and (23) together imply that the restratification associated with the TTW flow might be considered in terms of an effective buoyancy flux $\mathrm{EBF}_{g} \approx-0.05 H^{2}\left|\nabla_{h} b\right|^{2} /|f|$, similar to the scaling for the adiabatic buoyancy flux resulting from mixed layer instabilities from Fox-Kemper and Ferrari (2008). However, some caution is warranted in the direct comparison between the effect of these two processes on boundary layer restratification, as when averaged over the full boundary layer depth, the vertical structure of the MLI overturning circulation leads to $d N_{\mathrm{MLI}}^{2} / d t \approx$ $0.6\left(\nabla_{h} b\right)^{2} /|f|$. In contrast, the full TTW overturning circulation will be a function of the vertical and horizontal structure of the eddy viscosity and horizontal buoyancy gradient, as discussed in Wenegrat and McPhaden (2016). A simple estimate however comes from assuming vertically uniform $\nu$ and $\nabla_{h} b$, in which case $d N_{\mathrm{TTW}}^{2} / d t \sim 0.1\left|\nabla_{h} b\right|^{2} /|f|$, implying restratification over the full boundary layer should be largely dominated by MLIs.

A more complete way of comparing the different processes leading to restratification is given by (Lapeyre et al. 2006)

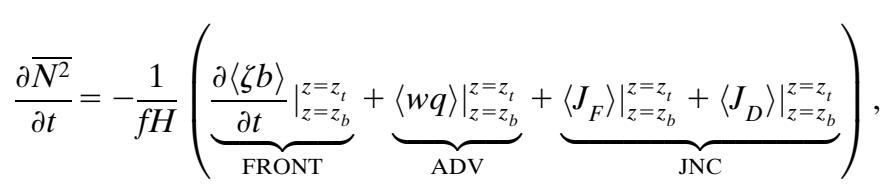

where $\overline{N^{2}}=\left\langle b\left(z=z_{t}\right)-b\left(z=z_{b}\right)\right\rangle / H$ is the bulk stratification, $H$ defines the distance between two depth levels $z_{t}$ and $z_{b}, \zeta$ is the relative vorticity, and the bracket notation indicates horizontal averaging. As discussed in Thomas and Ferrari (2008), terms on the right-hand side arise from the secondary circulations associated with surface frontogenesis (FRONT), vertical advection of potential vorticity (ADV), and nonconservative $\mathrm{J}$ vectors (JNC). As a first approximation, the first two terms can be identified with adiabatic instabilities, or other sources of secondary circulations (McWilliams 2017), which cause frontogenesis and advect high PV water from the thermocline into the boundary layer. The last terms can then be identified with nonconservative processes associated with boundary layer turbulence.

Plots of these quantities are shown in Fig. 15, for the model run discussed in section $3 \mathrm{~b}$. In the upper $75 \mathrm{~m}$ it can be seen that restratification is driven by a combination of both FRONT and JNC, with the periods of the largest rates of restratification (e.g., day $\sim 16$ ) dominated by nonconservative processes. In contrast, over the upper $150 \mathrm{~m}$, the initial mixed layer depth, restratification is dominated by ADV, associated with baroclinic instabilities lifting high PV water from the thermocline into the boundary layer. Although JNC is near zero for the first $\sim 20$ days, it should be noted that this term includes the effect of the constant surface buoyancy loss, which, if not offset by $\mathrm{EBF}_{g}$, would lead to significant destratification, as evident after day 35 (Fig. 15, bottom). The various processes dominating restratification during frontal spindown thus vary in time, and vertical position, with evidence that frictional restratification may be of leading-order importance near the surface.

Beyond the implications for restratification, the TTW circulations are also associated with significant PV fluxes (sections 3 and 4), which differ from adiabatic overturning circulations, which rearrange but do not change the volume-integrated PV. These processes are, however, closely coupled, as frontogenesis during the baroclinic instability process enhances the TTW PV flux, 

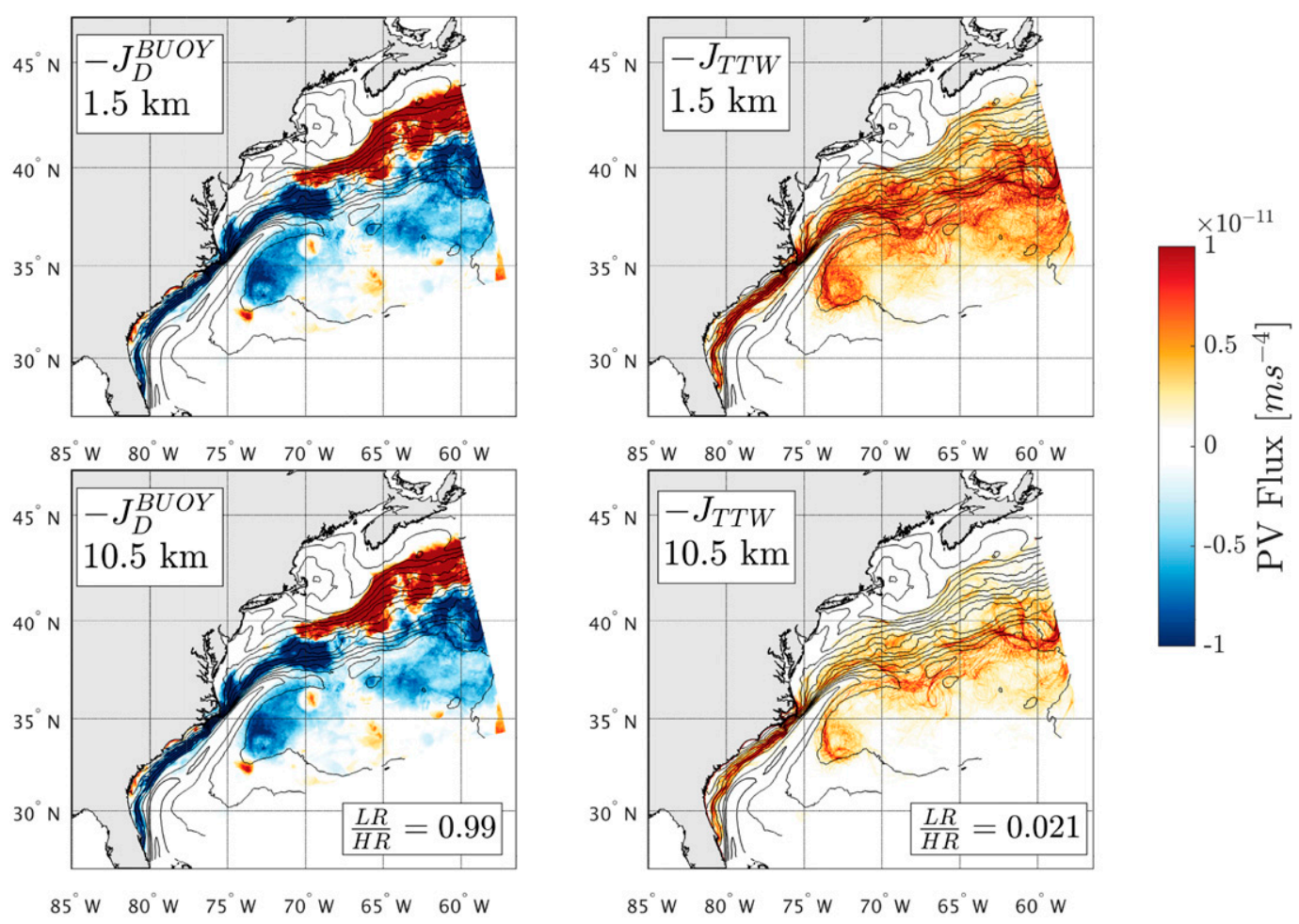

FIG. 14. Comparison of (left) surface buoyancy PV flux and (right) TTW PV flux evaluated (top) at high resolution (HR) and (bottom) using fields smoothed to lower resolution (LR). The ratio of the area-integrated scalings is indicated in the bottom plots.

in turn providing a boundary source of PV for the eddy equilibration process (Nakamura 1994). Models that do not resolve the submesoscale are thus likely to overestimate the net surface PV removal, with far-reaching implications, including for the seasonal cycle of mode water formation and destruction, as shown in section 4. Given the strong constraint PV provides on the general circulation, parameterization of these processes may be at least as important as parameterizing the restratifying effects of adiabatic submesoscale baroclinic instabilities. Implementation of a parameterization for the nonconservative effects discussed here would be simplified by the fact that the surface TTW flow is always down the buoyancy gradient, hence of the sense to inject PV, unlike wind-driven PV fluxes [(18)], which have a directional dependence, and hence require resolved submesoscale fronts.

\section{Summary}

In this manuscript we considered the flux of potential vorticity at the ocean surface and the importance of submesoscale processes in the surface boundary layer. Particular focus was given to the PV flux associated with the turbulent thermal wind, whereby boundary layer turbulence at a horizontal buoyancy gradient generates an ageostrophic cross-frontal circulation, leading to a downgradient flux of buoyancy, the geostrophic Ekman buoyancy flux [(9)]. The TTW flow thus leads to a source of PV at the ocean surface $J_{\text {TTW }}$, given by (17). Scaling arguments, developed in section 2 , and validated in section 3 , suggest that at the submesoscale $J_{\text {TTW }}$ can be of comparable magnitude to PV fluxes from the surface wind stress and surface buoyancy fluxes.

The frictional and diabatic PV fluxes are also shown to be coupled, with enhanced frictional PV fluxes largely offset by enhanced diabatic fluxes. This coupling between nonconservative terms in the momentum and buoyancy equations is consistent with realistic modeling studies that have noted that enhanced resolution of submesoscale processes does not always result in enhanced boundary layer restratification, as vertical buoyancy fluxes are partially compensated for by enhanced turbulent mixing (Capet et al. 2008). In appendix B we show this coupling of nonconservative PV fluxes formally using an asymptotic expansion, the results of which suggest that $\left|J_{F}^{\mathrm{TTW}}\right| \geq\left|J_{D}^{\mathrm{TTW}}\right|$; hence, the TTW balance is always a source of PV at the surface. Idealized modeling supports this analysis and validates simple scalings for the PV flux, which can be evaluated using observational data. 

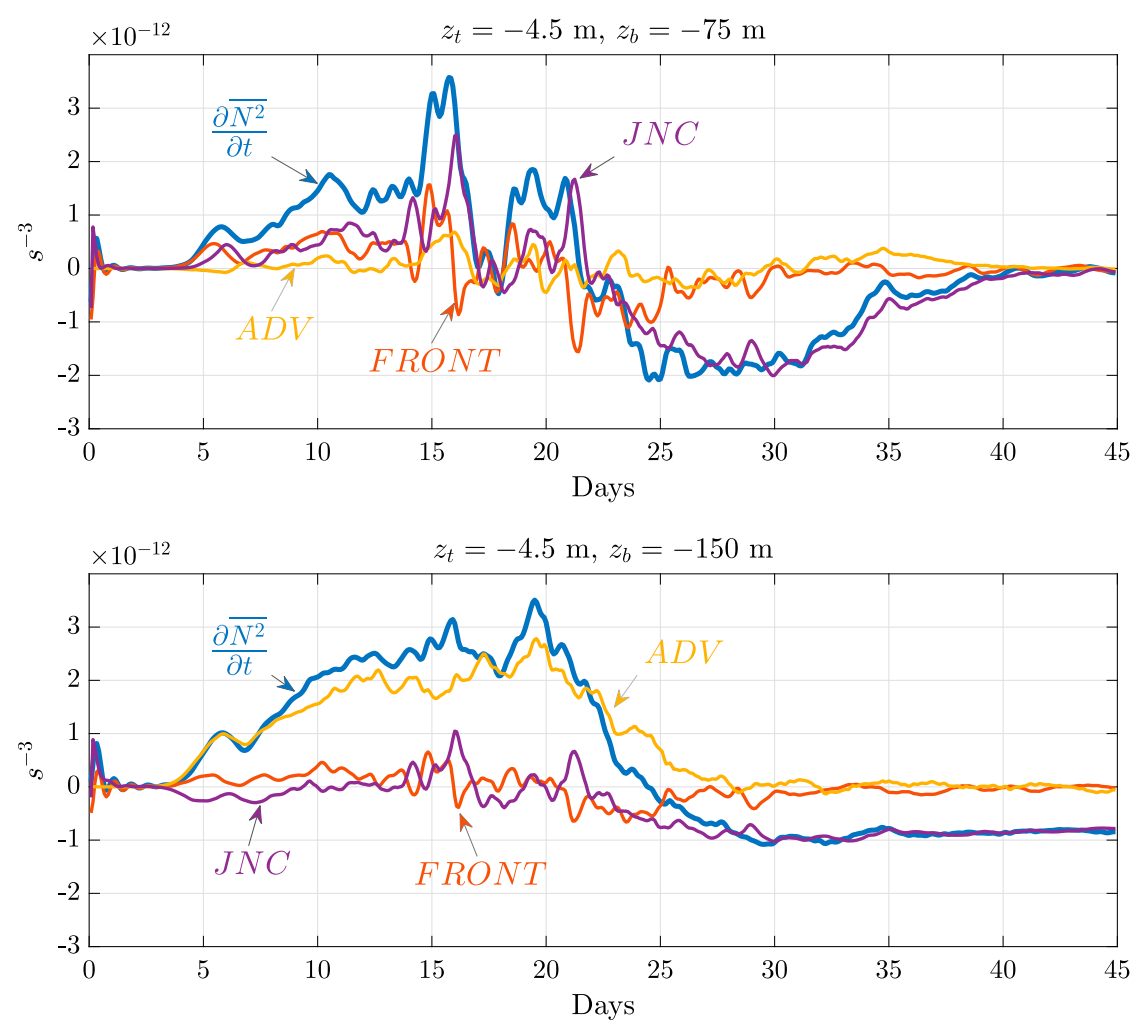

FIG. 15. Bulk stratification budget, as given by (25) and discussed in section 5, for the model run discussed in section $3 \mathrm{~b}\left[M_{o}^{2}=(2 f)^{2} \mathrm{~s}^{-2}, Q_{o}=25 \mathrm{~W} \mathrm{~m}^{-2}\right]$. Budget evaluated over the (top) upper 75 and (bottom) upper $150 \mathrm{~m}$.

Applying these scalings to a realistic model of the North Atlantic suggests that $J_{\text {TTW }}$ may be a leadingorder term in the PV budget of the Eighteen Degree Water, not accounted for in prior analyses using observations or lower-resolution numerical models. In the model, submesoscale processes reduce the rate of PV removal from the mode water by a factor of $\sim 2$ and shorten the formation season by approximately 3 weeks, leading to a cumulative seasonal change in mode water PV that is only $\sim 30 \%$ of that expected from surface buoyancy fluxes alone. The analysis here, performed at $1.5-\mathrm{km}$ resolution, likely provides a lower bound on the importance of submesoscale PV fluxes on the mode water PV budget. Likewise, deep mixed layers and strong horizontal buoyancy gradients predominate in mode water formation regions globally, and hence the conclusions drawn here regarding the importance of submesoscale processes in relation to the North Atlantic subtropical mode waters are potentially relevant to mode waters globally.

These findings thus serve to emphasize the role of the submesoscale in providing a direct connection between turbulence in the boundary layer and the properties of the gyre interior. Here we have focused on the modifications to the volume-integrated mode water $\mathrm{PV}$, which has consequences for the dynamics of the gyre-scale circulation through changes in the distribution, variability, and mixing of interior PV (Qiu et al. 2007). Important modifications to other physical and biogeochemical properties of the mode water by these processes are also anticipated. For example, in simulations of an idealized ocean gyre, Lévy et al. (2010) noted an increase in stratification of the mode water layer and a decrease of meridional heat transport when the submesoscale was resolved. The effect of these changes on biological productivity, and carbon export, will involve local changes to productivity in the boundary layer (Lévy et al. 2012a), gyre-scale responses to thermocline and nutricline depths (Lévy et al. 2012b), and preconditioning of the western boundary current source waters (Iudicone et al. 2016).

An important potential limitation to the generality of these findings arises from uncertainty in the properties of boundary layer turbulence at frontal systems, which will alter the effect of surface buoyancy forcing and $\mathrm{EBF}_{g}$ on the surface PV flux. For instance, using (7), it is evident that the two right-hand-side terms are directly related to the buoyancy production of turbulent kinetic energy and the geostrophic shear production of 
turbulent kinetic energy, respectively. Large-eddy simulations suggest that, under conditions of steady buoyancy loss conducive to the maintenance of forced symmetric instability, these terms may adjust such that, through $(8), J_{F}(0)+J_{D}(0) \approx f B_{o} / H$ (Taylor and Ferrari 2010; Bachman et al. 2017). This implies that, at least under particular dynamical conditions, changes to the structure of the vertical buoyancy flux and geostrophic shear production may lead to a total surface PV flux that is unchanged from classic upright convection. This type of behavior is evidently being captured to some degree by KPP (e.g., Fig. 7, left column); however, in these simulations PV injection at sharp fronts still dominates the area-integrated PV flux signal (see also Skyllingstad et al. 2017; Sullivan and McWilliams 2018). More generally, (7) reveals a direct link between the energetics of boundary layer turbulence and the surface flux of PV, providing a pathway for multiscale interaction and emphasizing the importance of improved understanding of how fronts modify boundary layer turbulence.

Finally, while adiabatic restratification by submesoscale baroclinic instabilities has been the subject of extensive work (Fox-Kemper et al. 2008; Fox-Kemper and Ferrari 2008; Fox-Kemper et al. 2011), nonconservative processes have received less attention. Here we show that boundary layer turbulence at submesoscale fronts can dominate the restratification of the near-surface layer (Fig. 15) and further lead to significant PV fluxes, crucial for setting the interior circulation. These frictional fluxes are not independent of the adiabatic baroclinic instabilities, but rather they are intertwined with the baroclinic instability process, being intensified by MLI frontogenesis, and in turn affecting the eddy life cycle by changing the PV of the boundary layer (Nakamura 1994). The PV source, and restratification, from the TTW circulation will be largely absent in coarsely resolved models, leading to a deficit of PV in the ocean interior.

Acknowledgments. The helpful comments and suggestions from John Marshall, George Nurser, and an anonymous reviewer are gratefully acknowledged. J.O.W. and L.N.T. were supported by NSF Grant OCE-1459677. J.C.M. and J.G. were supported by ONR N000141410626. ROMS simulations were performed using HPC resources from GENCI-TGCC (Grant 2017-A0010107638).

\section{APPENDIX A}

\section{PV Flux Near the Surface}

In section 2 the importance of the surface flux of PV was highlighted in light of the impermeability theorem [(6)]. However, a fundamental issue of concern is that the PV equation is higher order in spatial derivatives than the Navier-Stokes, indicating the potential difficulty of properly defining $J(0)$, as required if the volume integral in (6) is taken directly to the ocean surface. One potential strategy for introducing a surface boundary condition on the PV flux is the use of a singular delta sheet of boundary potential vorticity, as suggested by Schneider et al. (2003); however, this may not completely ameliorate issues related to the definition of $J(0)$. For example, if the turbulent stress follows MoninObukhov similarity theory, then near the surface $\nu \partial \mathbf{u} / \partial z$ is independent of $z$ and $\mathbf{F}=0$, implying that $J_{F}(0) \rightarrow 0$ as $z \rightarrow 0$. This suggests that the flux of PV at the surface will instead be governed by the departures from MoninObukhov similarity theory, and hence it will be sensitive to how the viscosity profile is regularized at the boundary. Introducing a small finite background viscosity/ diffusivity may be necessary for $J_{F}(0) \neq 0$, much as molecular viscosity effects are critical for the transfer of momentum and buoyancy fluxes within a very thin inner layer near the sea surface.

A simplifying result, relevant to the present investigation, can however be found under the assumption that some portion of the near-surface layer remains well mixed in buoyancy. In this case, the vertical PV flux will be approximately uniform over this layer, allowing for evaluation of the PV flux at some finite distance from the boundary, or as a vertically averaged quantity [as in (8)]. To see this, note that the PV flux near the surface can be written as

$$
J(z)=J(0)-\int_{z}^{0} \frac{\partial J}{\partial z} d z .
$$

Using (10), and assuming the horizontal buoyancy gradient is in the $x$ direction, the last term can be written as

$\int_{z}^{0} \frac{\partial J}{\partial z}=-\int_{z}^{0}\left(\omega_{a}^{z} \frac{\partial u_{i}}{\partial z} \frac{\partial b}{\partial x}+\omega_{a}^{z} \frac{\partial}{\partial z} w \frac{\partial b}{\partial z}-\frac{\partial w q}{\partial z}\right) d z$,

where the vertical integration is understood to be taken over the well-mixed portion of the surface boundary layer, such that $\partial^{2} b / \partial t \partial z=0$. The relative contribution of terms on the right-hand side of (A1) can then be determined by employing representative scales $x \sim L$, $z \sim h, t \sim f^{-1}, u_{i} \sim \operatorname{Ro} U, w_{i} \sim \operatorname{Ro} U H / L, b \sim \beta$, and by assuming that vertical derivatives scale with $H$. The scaling for the velocity comes from assuming balanced dynamics and noting that to leading order in Rossby number, $\mathrm{Ro}=U / f L$, the inviscid flow is in thermal wind balance, that is, aligned along buoyancy contours; hence, the sheared cross-frontal velocity enters at $O(\mathrm{Ro})$. Next, scaling each term in (A2) relative to the surface PV flux, 
which by (10) goes as $J(0) \sim f \partial b / \partial t \sim f^{2} \beta$, gives that all terms in $(\mathrm{A} 2)$ are $O\left(\operatorname{Ro}^{2} h / H\right)$. Thus, for $\operatorname{Ro}^{2} \ll 1$, or $h / H \ll 1$, the vertical PV flux will be approximately constant over the well-mixed portion of the boundary layer,

$$
J(z) \approx J(0), \text { for } z>-h .
$$

A similar result was found in the low Rossby number limit by Marshall and Nurser (1992) and in the case of resolved turbulent fluxes by Taylor and Ferrari (2010).

It is also useful to note that since $w q=0$ at $z=0$, it also follows directly from the abovementioned scaling arguments that the total near-surface PV flux will be dominated by the nonadvective component of the flux. Vertically integrating the nonadvective PV flux can thus be used to express the surface flux in terms of averaged quantities, as in (8).

\section{APPENDIX B}

\section{Asymptotic Analysis of TTW PV Fluxes}

As discussed in section 2, the TTW circulation is restratifying, and hence is expected, through (8) and (10), to lead to a net downward flux of PV at the surface. The magnitude of the total TTW PV flux will however be the residual of partial cancellation between the frictional PV injection (through TTW advection of the horizontal buoyancy gradient) and the diabatic PV removal as a result of the turbulent mixing of buoyancy. In this appendix we provide an asymptotic analysis of an idealized frontal configuration, to illustrate the coupling between across-front advection and turbulent mixing, explaining the scalings used in (19) and (20).

We will focus the asymptotic analysis on the upper limb of the TTW circulation, which allows considerable simplification of the problem, isolating the essential dynamics. This approximation is also consistent with numerical solutions of idealized TTW problems, which show that the TTW circulation will initially tilt a finite-width front in the horizontal, offsetting spatially the upper and lower limbs of the secondary circulation. At the end of the appendix, the full overturning circulation is considered using numerical solutions. We therefore first consider an idealized front with a vertically and horizontally uniform horizontal buoyancy gradient in the $x$ direction, and no variations in the $y$ direction. We ignore horizontal mixing $\left(\kappa_{h} / \kappa \ll L^{2} / H^{2}\right)$, and assume that the viscosity $\nu$ and diffusivity $\kappa$ are constants. Variables are nondimensionalized as $(u, v)=U\left(u^{\prime}, v^{\prime}\right), z=H z^{\prime}$, $x=(U / f) x^{\prime}, b=\left(U \overline{M^{2}} / f\right) b^{\prime}$, and $p=\left(U \overline{M^{2}} h / f\right) p^{\prime}$, where $\overline{M^{2}}$ is an imposed external scale for the horizontal buoyancy gradient. Time is nondimensionalized using the stratified spinup time scale $t=E^{-1 / 2} f^{-1} t^{\prime}$, where $E=2 \nu /\left(f H^{2}\right)=\delta_{e}^{2} / H^{2}$ is the Ekman number (Greenspan and Howard 1963). The relevant governing equations are thus

$$
\begin{aligned}
E^{1 / 2} \frac{\partial u^{\prime}}{\partial t^{\prime}}-v^{\prime} & =-\frac{\partial p^{\prime}}{\partial x^{\prime}}+\frac{E}{2} \frac{\partial^{2} u^{\prime}}{\partial z^{\prime 2}}, \\
E^{1 / 2} \frac{\partial v^{\prime}}{\partial t^{\prime}}+u^{\prime} & =\frac{E}{2} \frac{\partial^{2} v^{\prime}}{\partial z^{\prime 2}}, \\
0 & =-\frac{\partial p^{\prime}}{\partial z^{\prime}}+b^{\prime}, \quad \text { and } \\
E^{1 / 2} \frac{\partial b^{\prime}}{\partial t^{\prime}}+u^{\prime} M^{\prime 2} & =\frac{E}{2} \frac{\partial^{2} b^{\prime}}{\partial z^{\prime 2}} .
\end{aligned}
$$

In the equations given above $M^{2}=\partial b^{\prime} / \partial x^{\prime}$, and we have assumed $\nu / \kappa=1$. For simplicity we consider the case of no surface wind stress and no surface buoyancy flux, although these restrictions can be relaxed (as in Thomas and Rhines 2002). Given these assumptions, the surface boundary conditions are

$$
\begin{aligned}
& \frac{\partial u^{\prime}}{\partial z^{\prime}}=\frac{\partial v^{\prime}}{\partial z^{\prime}}=0 \quad \text { at } \quad z^{\prime}=0, \quad \text { and } \\
& \frac{\partial b^{\prime}}{\partial z^{\prime}}=0 \quad \text { at } \quad z^{\prime}=0 .
\end{aligned}
$$

Consistent with the discussion above, we will look for solutions that decay away from the surface, excluding the lower limb of the TTW circulation (Wenegrat and McPhaden 2016).

In the interest of notational simplicity, we forgo primes for the remainder of the appendix. Following Thomas and Rhines (2002), the nondimensionalized variables are expanded in terms of interior, thermal, and Ekman layers (subscripts $i, t$, and $e$, respectively) as

$$
\begin{aligned}
& u=u_{i}(z, t)+u_{t}(\xi, t)+u_{e}(\eta, t), \\
& v=v_{i}(z, t)+v_{t}(\xi, t)+v_{e}(\eta, t), \quad \text { and } \\
& b=b_{i}(z, t)+b_{t}(\xi, t)+b_{e}(\eta, t),
\end{aligned}
$$

where $\xi=E^{-1 / 4} z$ and $\eta=E^{-1 / 2} z$. A perturbation expansion is then performed in powers of the Ekman number, that is, $X=\sum_{n=0}^{\infty} E^{n / 4} X^{(n)}$.

\section{a. Momentum equations}

To leading order the interior velocity solutions are in geostrophic balance,

$$
\begin{aligned}
& v_{i}^{(0)}=\frac{\partial p}{\partial x}, \quad \text { and } \\
& u_{i}^{(0)}=0 .
\end{aligned}
$$


In the thermal boundary layer,

$$
\begin{aligned}
& E^{1 / 2} \frac{\partial u_{t}}{\partial t}-v_{t}=E^{1 / 2} \frac{1}{2} \frac{\partial^{2} u_{t}}{\partial \xi^{2}}, \quad \text { and } \\
& E^{1 / 2} \frac{\partial v_{t}}{\partial t}+u_{t}=E^{1 / 2} \frac{1}{2} \frac{\partial^{2} v_{t}}{\partial \xi^{2}} .
\end{aligned}
$$

Thus, for this frontal zone configuration, $u_{t}=v_{t}=0$, as there is no term available at any order to balance the Coriolis acceleration. In the more general case, where horizontally varying buoyancy anomalies develop in the thermal boundary layer, an $O\left(E^{1 / 4}\right)$ velocity correction in thermal wind balance can develop (Thomas and Rhines 2002).

In the Ekman layer, the alongfront momentum balance is

$$
E^{1 / 2} \frac{\partial v_{e}}{\partial t}+u_{e}=\frac{1}{2} \frac{\partial^{2} v_{e}}{\partial \eta^{2}} .
$$

Without a surface wind stress, the leading-order Ekman layer solution is at $O\left(E^{1 / 2}\right)$, which can be seen from the surface boundary condition [(B5)], such that

$$
\begin{aligned}
& \frac{\partial v_{e}^{(2)}}{\partial \eta}=-\frac{\partial v_{i}^{(0)}}{\partial z}, \quad \text { at } \quad z=0, \quad \text { and } \\
& \frac{\partial u_{e}^{(2)}}{\partial \eta}=0, \quad \text { at } \quad z=0 .
\end{aligned}
$$

The relevant solution for cross-front velocity is therefore the TTW solution (McWilliams et al. 2015)

$$
u_{e}^{(2)}=-\frac{1}{2} M^{2} e^{\eta}[\cos (\eta)-\sin (\eta)] .
$$

Thus, to leading order, the frictional PV flux in the Ekman layer is

$$
J_{F_{e}}^{\mathrm{TTW}}=E^{1 / 2} u_{e}^{(2)}(0) M^{2}=-\frac{1}{2} E^{1 / 2} M^{4} .
$$

The right-hand side is always negative; hence, there is always a frictional injection of PV in the Ekman layer as a result of the TTW flow. This injection will however be partially compensated for by diabatic removal, as shown below.

\section{b. Buoyancy equations}

The first interior buoyancy solution is a balance between the tendency of the $O\left(E^{1 / 2}\right)$ buoyancy with the diffusion of the zeroth-order interior buoyancy, which we assume is negligible. The buoyancy equation in the Ekman layer is

$$
E^{1 / 2} \frac{\partial b_{e}}{\partial t}+u_{e} M^{2}=\frac{1}{2} \frac{\partial^{2} b_{e}}{\partial \eta^{2}}
$$

The first nonzero solution for buoyancy in the Ekman layer will therefore be at $O\left(E^{1 / 2}\right)$,

$$
u_{e}^{(2)} M^{2}=\frac{1}{2} \frac{\partial^{2} b_{e}^{(2)}}{\partial \eta^{2}} .
$$

Thus, in the Ekman layer, the horizontal Ekman flux of buoyancy is balanced by turbulent mixing of buoyancy, which leads to a diabatic flux of PV. To leading order, this PV flux can be written as

$$
J_{D_{e}}^{\mathrm{TTW}}=\frac{1}{2} E^{1 / 2} M^{4}=-J_{F_{e}}^{\mathrm{TTW}},
$$

which exactly cancels the frictional PV injection in the Ekman layer [(B18)].

This balance in the Ekman layer is however coupled to the buoyancy equation in the thermal boundary layer through the surface boundary condition, which can be seen by vertically integrating (B20),

$$
U_{e} M^{2}=\left.\frac{1}{2} \frac{\partial b_{e}^{(2)}}{\partial \eta}\right|_{z=0}
$$

where $U_{e}=\int_{-\infty}^{0} u_{e}^{(2)} d \eta$. The cross-frontal TTW advection thus acts as an effective buoyancy flux, the geostrophic Ekman buoyancy flux (Bachman and Taylor 2016),

$$
\mathrm{EBF}_{g}^{\prime}=E^{1 / 2} U_{e} M^{2},
$$

where we retain the prime to emphasize the nondimensional form. The surface boundary condition on the buoyancy equation [(B6)] can therefore be written as

$$
\frac{\partial b_{t}}{\partial \xi}=-2 E^{-1 / 4} \mathrm{EBF}_{g}^{\prime}, \quad \xi=0,
$$

where we have assumed for simplicity that there is no surface buoyancy flux, and again that the interior stratification is small.

The relevant buoyancy equation in the thermal boundary layer [at $O\left(E^{1 / 4}\right)$ ] is

$$
\frac{\partial b_{t}^{(1)}}{\partial t}=\frac{1}{2} \frac{\partial^{2} b_{t}^{(1)}}{\partial \xi^{2}} .
$$

The solution for $b_{t}$, subject to the boundary condition given by (B24), and an assumption of no initial buoyancy anomaly, is given by

$$
b_{t}=-2 E^{-1 / 4} \operatorname{EBF}_{g}^{\prime} \sqrt{2 t} \int_{-\xi / \sqrt{2 t}}^{\infty} \operatorname{erfc}(s) d s,
$$

where $\operatorname{erfc}(s)$ is the complementary error function (Abramowitz and Stegun 1964). The vertical diabatic 

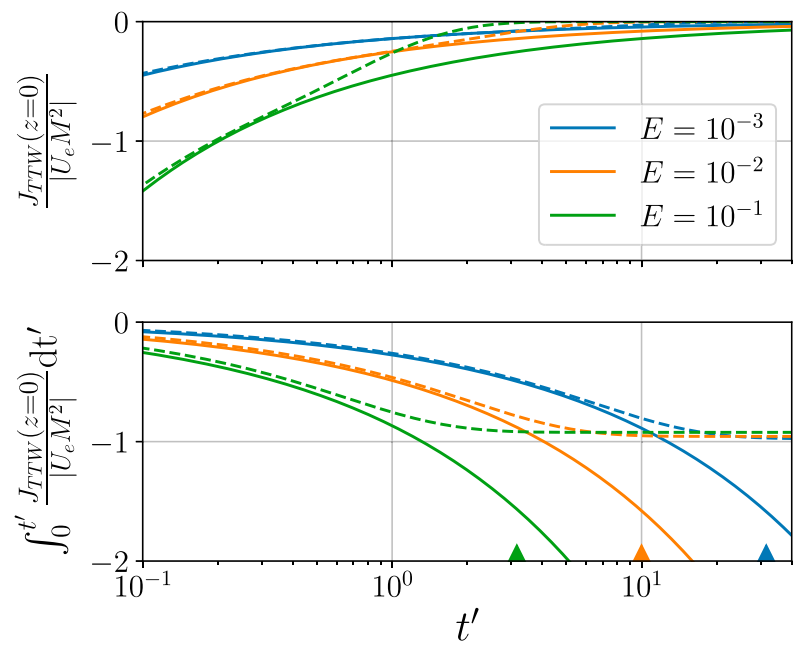

FIG. B1. (top) Nondimensional surface PV flux and (bottom) time-integrated cumulative surface PV flux, for the simplified model discussed in appendix B, as a function of Ekman number (legend). Analytical solutions for a semi-infinite domain [solid lines; (B28)] and numerical solutions for a finite depth layer, including $\mathrm{EBF}_{g}$ at the base of the layer as a result of the lower limb of the TTW circulation (dashed lines). In each case, as the diffusive boundary layer deepens relative to the Ekman layer, the magnitude of the PV flux decreases. However, the cumulative PV flux in the bottom panel is always oriented downward, hence always of the sense to increase the $\mathrm{PV}$. The vertical diffusion time scale, $\tau_{v} \sim(E f)^{-1}$, for each Ekman number is indicated by the arrow markers in the bottom panel. All quantities are nondimensionalized as in appendix $\mathrm{B}$, with $t^{\prime}=E^{1 / 2} f t$ giving the nondimensional Ekman spindown time scale.

PV flux at the surface, for the thermal boundary layer, is thus

$$
J_{D_{t}}^{\mathrm{TTW}}(\xi=0)=E^{-1 / 4} \mathrm{EBF}_{g}^{\prime} \sqrt{\frac{2}{\pi t}},
$$

and the total TTW PV flux can be written as

$$
J_{F}^{\mathrm{TTW}}+J_{D}^{\mathrm{TTW}}=J_{F_{e}}^{\mathrm{TTW}}+J_{D_{e}}^{\mathrm{TTW}}+J_{D_{t}}^{\mathrm{TTW}} \approx \frac{\mathrm{EBF}_{g}^{\prime}}{h\left(t^{\prime}\right)}
$$

where $h\left(t^{\prime}\right)=E^{1 / 4} \sqrt{\pi t^{\prime} / 2}$ is the time-varying thermal boundary layer depth and the primes are included to emphasize the nondimensional form. The TTW PV flux can therefore be conceptualized in terms of an effective buoyancy flux that generates a positive surface buoyancy tendency $\left(\partial b /\left.\partial t\right|_{z=0}>0\right)$ that decreases in time as vertical mixing diffuses the buoyancy flux over an increasingly deep layer (Fig. B1). Similar results are evident in the analysis of Young (1994).

The analysis stated above can easily be extended to a layer of finite depth by adding no-flux boundary conditions on momentum and buoyancy at $z^{\prime}=-1$. In this case the lower limb of the TTW circulation also generates a geostrophic Ekman buoyancy flux, such that (B24) gives both the surface and lower boundary conditions, and solutions to (B25) can be found using standard series methods. In this idealized frontal zone configuration, the negative buoyancy source from the lower limb of the TTW circulation will exactly offset the positive buoyancy source associated with the upper limb of the overturning circulation, and equilibrium solutions are thus possible. In more realistic configurations, where $\int_{-H}^{0} \mathbf{u}_{e}^{\mathrm{TTW}} \cdot \nabla_{h} b d z \neq 0$, this will not be the case. It is also worth noting that horizontal mixing can also arrest TTW frontogenesis (McWilliams et al. 2015), which in the context of PV dynamics can be understood as a time-dependent process whereby the frictional injection of PV is intensified through surface frontogenesis but is ultimately offset by the growing diabatic contribution as the horizontal length scale shrinks. However, regardless of the possible existence of equilibrium solutions, there will still be a cumulative source of PV at the surface from the TTW circulation, as the equilibration process does not occur until turbulence has adjusted the boundary layer vertically (horizontally) over a time scale $\tau_{v} \sim H^{2} / \kappa\left(\tau_{h} \sim L^{2} / \kappa_{h}\right)$, as shown in Fig. B1.

\section{APPENDIX C}

\section{Configuration of the MITgcm}

The most relevant aspects of the model configuration are documented in section 3; however, in the interest of reproducibility, further details of the model configuration are documented here.

For all runs, horizontal mixing of momentum is parameterized using a biharmonic operator, with a Smagorinsky coefficient of 3, and Leith and modified Leith coefficients of 1 (as in Brannigan et al. 2015). Horizontal diffusion of temperature is implemented using a uniform biharmonic diffusivity of $\kappa_{H}=10^{6} \mathrm{~m}^{4} \mathrm{~s}^{-1}$. The Prather advection scheme (Prather 1986), a secondorder moment scheme that has been shown to limit numerical diffusion (Hill et al. 2012), is used for temperature advection. These values were found by experimentation to provide a good balance of not being overly diffusive while still minimizing implicit numerical diffusion.

As noted above, the KPP scheme is used for vertical mixing; however, it is worth documenting that in the pure convective conditions considered here, we found it necessary to ensure that the package configuration option for horizontal smoothing of diffusivity/viscosity was disabled and that vertical smoothing of the Richardson number was enabled. Without these choices, the strong Ekman restratification at sharp fronts would occasionally lead to very thin KPP boundary layer depths overlying unstable density profiles that could persist for long periods of time. A possible 
interpretation of this is that the horizontal smoothing of turbulence properties across multiple grid cells is physically inconsistent in the presence of sharp frontal features with horizontal scales approaching the grid scale.

The model is initialized in a geostrophically balanced double-front configuration, with the initial buoyancy field given by

$$
b_{o}= \begin{cases}\Delta b_{o} Y(y) Z(z), & \text { if } z \geq-H_{o} \\ \Delta b_{o} Y(y) Z(z)+N_{\mathrm{INT}}^{2}\left(z+H_{o}\right), & \text { otherwise, }\end{cases}
$$

where $\Delta b_{o}$ is the change in buoyancy across the front such that $\Delta b_{o} / L_{f}=M_{o}^{2}$, and

$$
Y(y)=\left\{\begin{array}{ll}
0.5\left[1-\tanh \left(\frac{y}{L_{f}}\right)+\tanh \left(\frac{y-L_{y} / 2}{L_{f}}\right)\right], & 0 \leq y \leq L_{y} / 2, \quad \\
0.5\left[\tanh \left(\frac{y-L_{y} / 2}{L_{f}}\right)-\tanh \left(\frac{y-L_{y}}{L_{f}}\right)-1\right], & L_{y} / 2 \leq y \leq L_{y}
\end{array} \quad\right. \text { and }
$$

$$
Z(z)=0.5\left[\tanh \left(\frac{z+1.5 H_{o}}{0.15 H_{o}}\right)+1\right]
$$

To avoid spurious diabatic removal of PV as a result of the bottom no-flux boundary condition, the bottom two grid cells were set to have equal buoyancy. The numerical values of all the parameters above are given in Table 1. The growth rate of MLIs is dependent on the initial horizontal buoyancy gradient (Boccaletti et al. 2007). Thus, to allow sufficient time for a well-developed turbulence field to develop in each case, we run the $M_{o}^{2}=(1 f)^{2}$ runs for 60 days, the $M_{o}^{2}=(2 f)^{2}$ runs for 45 days, and the remaining runs for 30 days.

The potential vorticity budgets were constructed offline, using time-averaged diagnostics output at 2-h time steps. The nonconservative $\mathrm{J}$ vectors were constructed using the built-in MITgcm diagnostics for the temperature [(4)] and momentum budgets [(5)]. The exception to this being the advection terms in each budget, which were reconstructed offline using velocity diagnostics and a second-order accurate derivative operator. This approach allows numerical diffusion and viscosity to be approximated as the difference between the online and offline advection diagnostic in each budget. These terms are generally small, with some transient exceptions at particularly sharp frontal features.

Practically, for evaluating the vertical PV fluxes in gridded numerical models, it is necessary to evaluate the vertical fluxes at a finite depth, $z=-\Delta z$, where $\Delta z$ will depend on the vertical resolution. The impermeability theorem can still be applied in this case, but it formally requires adding the vertical advective flux of $\mathrm{PV}$ to the right-hand side of (6). However, in a well-mixed boundary layer, and at small $\Delta z$, both $q$ and $w$ are small; hence, (6) remains an accurate approximation, as discussed in appendix A. In the simulations of section 3, a minimum boundary layer depth of $12 \mathrm{~m}$ was set for KPP, ensuring that at least four $\rho$ points were in the boundary layer at all times. This approach proved sufficiently accurate for closing the PV budgets for the destabilizing surface forcing we consider; however, simulations with shallower boundary layers, or stabilizing forcing, might require additional near-surface resolution.

\section{REFERENCES}

Abramowitz, M., and I. A. Stegun, Eds., 1964: Handbook of Mathematical Functions: With Formulas, Graphs, and Mathematical Tables. Dover Books on Mathematics, Dover Publications, $1046 \mathrm{pp}$.

Bachman, S. D., and J. R. Taylor, 2016: Numerical simulations of the equilibrium between eddy-induced restratification and vertical mixing. J. Phys. Oceanogr., 46, 919-935, https:// doi.org/10.1175/JPO-D-15-0110.1.

— , B. Fox-Kemper, J. Taylor, and L. Thomas, 2017: Parameterization of frontal symmetric instabilities. I: Theory for resolved fronts. Ocean Modell., 109, 72-95, https://doi.org/ 10.1016/j.ocemod.2016.12.003.

Bates, N. R., A. C. Pequignet, R. J. Johnson, and N. Gruber, 2002: A short-term sink for atmospheric $\mathrm{CO}_{2}$ in subtropical mode water of the North Atlantic Ocean. Nature, 420, 489-493, https://doi.org/10.1038/nature01253.

Billheimer, S., and L. D. Talley, 2016: Annual cycle and destruction of Eighteen Degree Water. J. Geophys. Res. Oceans, 121, 6604-6617, https://doi.org/10.1002/2016JC011799.

Boccaletti, G., R. Ferrari, and B. Fox-Kemper, 2007: Mixed layer instabilities and restratification. J. Phys. Oceanogr., 37, 22282250, https://doi.org/10.1175/JPO3101.1.

Brannigan, L., D. P. Marshall, A. Naveira-Garabato, and A. George Nurser, 2015: The seasonal cycle of submesoscale flows. Ocean Modell., 92, 69-84, https://doi.org/ 10.1016/j.ocemod.2015.05.002.

Callies, J., R. Ferrari, J. M. Klymak, and J. Gula, 2015: Seasonality in submesoscale turbulence. Nat. Commun., 6, 6862, https:// doi.org/10.1038/ncomms7862.

Capet, X., J. C. McWilliams, M. J. Molemaker, and A. F. Shchepetkin, 2008: Mesoscale to submesoscale transition in the California Current System. Part I: Flow structure, eddy 
flux, and observational tests. J. Phys. Oceanogr., 38, 29-43, https://doi.org/10.1175/2007JPO3671.1.

Cronin, M. F., and W. S. Kessler, 2009: Near-surface shear flow in the tropical Pacific cold tongue front. J. Phys. Oceanogr., 39, 1200-1215, https://doi.org/10.1175/2008JPO4064.1.

D'Asaro, E., C. Lee, L. Rainville, R. Harcourt, and L. Thomas, 2011: Enhanced turbulence and energy dissipation at ocean fronts. Science, 332, 318-322, https://doi.org/10.1126/science.1201515.

Deremble, B., and W. K. Dewar, 2012: First-order scaling law for potential vorticity extraction due to wind. J. Phys. Oceanogr., 42, 1303-1312, https://doi.org/10.1175/JPO-D-11-0136.1.

_- and —, 2013: Volume and potential vorticity budgets of Eighteen Degree Water volume. J. Phys. Oceanogr., 43, 23092321, https://doi.org/10.1175/JPO-D-13-052.1.

— , N. Wienders, and W. K. Dewar, 2014: Potential vorticity budgets in the North Atlantic Ocean. J. Phys. Oceanogr., 44, 164-178, https://doi.org/10.1175/JPO-D-13-087.1.

Forget, G., G. Maze, M. Buckley, and J. Marshall, 2011: Estimated North Atlantic Eighteen Degree Water volume. J. Phys. Oceanogr., 41, 269-286, https://doi.org/10.1175/ 2010JPO4257.1.

Fox-Kemper, B., and R. Ferrari, 2008: Parameterization of mixed layer eddies. Part II: Prognosis and impact. J. Phys. Oceanogr., 38, 1166-1179, https://doi.org/10.1175/2007JPO3788.1.

,$- \ldots$, and R. Hallberg, 2008: Parameterization of mixed layer eddies. Part I: Theory and diagnosis. J. Phys. Oceanogr., 38, 1145-1165, https://doi.org/10.1175/2007JPO3792.1.

_ _ and Coauthors, 2011: Parameterization of mixed layer eddies. III: Implementation and impact in global ocean climate simulations. Ocean Modell., 39, 61-78, https://doi.org/ 10.1016/j.ocemod.2010.09.002.

Greenspan, H. P., and L. N. Howard, 1963: On a time-dependent motion of a rotating fluid. J. Fluid Mech., 17, 385-404, https:// doi.org/10.1017/S0022112063001415.

Gula, J., M. J. Molemaker, and J. C. McWilliams, 2014: Submesoscale cold filaments in the Gulf Stream. J. Phys. Oceanogr., 44, 2617-2643, https://doi.org/10.1175/JPO-D-14-0029.1. , -, and - , 2015: Gulf Stream dynamics along the southeastern U.S. seaboard. J. Phys. Oceanogr., 45, 690-715, https://doi.org/10.1175/JPO-D-14-0154.1.

Hanawa, K., and L. D. Talley, 2001: Mode waters. Ocean Circulation and Climate: Observing and Modelling the Global Ocean, International Geophysics Series, Vol. 77, Academic Press, 373386, https://doi.org/10.1016/S0074-6142(01)80129-7.

Haynes, P. H., and M. E. McIntyre, 1987: On the evolution of vorticity and potential vorticity in the presence of diabatic heating and frictional or other forces. J. Atmos. Sci., 44, 828-841, https://doi.org/10.1175/1520-0469(1987) 044<0828:OTEOVA $>2.0 . \mathrm{CO} ; 2$.

Hill, C., D. Ferreira, J.-M. Campin, J. Marshall, R. Abernathey, and N. Barrier, 2012: Controlling spurious diapycnal mixing in eddy-resolving height-coordinate ocean modelsInsights from virtual deliberate tracer release experiments. Ocean Modell., 45-46, 14-26. https://doi.org/10.1016/ j.ocemod.2011.12.001.

Iudicone, D., K. B. Rodgers, Y. Plancherel, O. Aumont, T. Ito, R. M. Key, G. Madec, and M. Ishii, 2016: The formation of the ocean's anthropogenic carbon reservoir. Sci. Rep., 6, https:// doi.org/10.1038/srep35473.

Kelly, K. A., and S. Dong, 2013: The contributions of atmosphere and ocean to North Atlantic Subtropical Mode Water volume anomalies. Deep-Sea Res. II, 91, 111-127, https://doi.org/ 10.1016/j.dsr2.2013.02.020.
Keyser, D., and R. Rotunno, 1990: On the formation of potentialvorticity anomalies in upper-level jet-front systems. Mon. Wea. Rev., 118, 1914-1921, https://doi.org/10.1175/1520-0493(1990) $118<1914$ :OTFOPV $>2.0$.CO;2.

Kraus, E. B., and J. A. Businger, 1994: Atmosphere-Ocean Interaction. 2nd ed. Oxford Monogr. Geol. Geophys., No. 27, Clarendon Press, 384 pp.

Kwon, Y.-O., and S. C. Riser, 2004: North Atlantic Subtropical Mode Water: A history of ocean-atmosphere interaction 1961-2000. Geophys. Res. Lett., 31, L19307, https://doi.org/ 10.1029/2004GL021116.

Lapeyre, G., P. Klein, and B. L. Hua, 2006: Oceanic restratification forced by surface frontogenesis. J. Phys. Oceanogr., 36, 15771590, https://doi.org/10.1175/JPO2923.1.

Large, W. G., J. C. McWilliams, and S. C. Doney, 1994: Oceanic vertical mixing: A review and a model with a nonlocal boundary layer parameterization. Rev. Geophys., 32, 363-403, https://doi.org/10.1029/94RG01872.

Lévy, M., P. Klein, A.-M. Tréguier, D. Iovino, G. Madec, S. Masson, and K. Takahashi, 2010: Modifications of gyre circulation by sub-mesoscale physics. Ocean Modell., 34, 1-15, https://doi.org/10.1016/j.ocemod.2010.04.001.

_ R. Ferrari, P. J. S. Franks, A. P. Martin, and P. Rivière, 2012a: Bringing physics to life at the submesoscale. Geophys. Res. Lett., 39, L14602, https://doi.org/10.1029/2012GL052756.

— , D. Iovino, L. Resplandy, P. Klein, G. Madec, A.-M. Tréguier, S. Masson, and K. Takahashi, 2012b: Large-scale impacts of submesoscale dynamics on phytoplankton: Local and remote effects. Ocean Modell., 43-44, 77-93, https://doi.org/10.1016/ j.ocemod.2011.12.003.

Mahadevan, A., E. D'Asaro, C. Lee, and M. J. Perry, 2012: Eddydriven stratification initiates North Atlantic spring phytoplankton blooms. Science, 337, 54-58, https://doi.org/10.1126/ science. 1218740 .

Marshall, J. C., and A. J. G. Nurser, 1992: Fluid dynamics of oceanic thermocline ventilation. J. Phys. Oceanogr., 22, 583-595, https://doi.org/10.1175/1520-0485(1992)022<0583: FDOOTV $>2.0 . \mathrm{CO} ; 2$.

— C. Hill, L. Perelman, and A. Adcroft, 1997: Hydrostatic, quasihydrostatic, and nonhydrostatic ocean modeling. J. Geophys. Res., 102, 5733-5752, https://doi.org/10.1029/96JC02776.

__ D. Jamous, and J. Nilsson, 2001: Entry, flux, and exit of potential vorticity in ocean circulation. J. Phys. Oceanogr., 31, 777-789, https://doi.org/10.1175/1520-0485(2001)031<0777: EFAEOP $>2.0 . \mathrm{CO} ; 2$.

Maze, G., and J. Marshall, 2011: Diagnosing the observed seasonal cycle of Atlantic Subtropical Mode Water using potential vorticity and its attendant theorems. J. Phys. Oceanogr., 41, 1986-1999, https://doi.org/10.1175/2011JPO4576.1.

_ , J. Deshayes, J. Marshall, A.-M. Tréguier, A. Chronis, and L. Vollmer, 2013: Surface vertical PV fluxes and subtropical mode water formation in an eddy-resolving numerical simulation. Deep-Sea Res. II, 91, 128-138, https://doi.org/10.1016/ j.dsr2.2013.02.026.

McCartney, M. S., 1982: The subtropical recirculation of mode waters. J. Mar. Res., 40, 427-464.

McIntyre, M. E., and W. A. Norton, 1990: Dissipative wave-mean interactions and the transport of vorticity or potential vorticity. J. Fluid Mech., 212, 403-435, https://doi.org/10.1017/ S0022112090002038.

McWilliams, J. C., 2016: Submesoscale currents in the ocean. Proc. Roy. Soc. London, 472A, 20160117, https://doi.org/10.1098/ rspa.2016.0117. 
2017: Submesoscale surface fronts and filaments: Secondary circulation, buoyancy flux, and frontogenesis. J. Fluid Mech., 823, 391-432, https://doi.org/10.1017/jfm.2017.294.

_ J. J. Gula, M. J. Molemaker, L. Renault, and A. F. Shchepetkin, 2015: Filament frontogenesis by boundary layer turbulence. J. Phys. Oceanogr., 45, 1988-2005, https://doi.org/10.1175/ JPO-D-14-0211.1.

Nakamura, N., 1994: Nonlinear equilibration of two-dimensional Eady waves: Simulations with viscous geostrophic momentum equations. J. Atmos. Sci., 51, 1023-1035, https://doi.org/ 10.1175/1520-0469(1994)051<1023:NEOTDE > 2.0.CO;2.

Nurser, A. J. G., and J. C. Marshall, 1991: On the relationship between subduction rates and diabatic forcing of the mixed layer. J. Phys. Oceanogr., 21, 1793-1802, https://doi.org/ 10.1175/1520-0485(1991)021<1793:OTRBSR > 2.0.CO;2.

Prather, M. J., 1986: Numerical advection by conservation of secondorder moments. J. Geophys. Res., 91, 6671-6681, https://doi.org/ 10.1029/JD091iD06p06671.

Qiu, B., P. Hacker, S. Chen, K. Donohue, D. Watts, H. Mitsudera, N. Hogg, and S. Jayne, 2006: Observations of the subtropical mode water evolution from the Kuroshio Extension. J. Phys. Oceanogr., 36, 457-473, https://doi.org/10.1175/JPO2849.1.

_ S. Chen, and P. Hacker, 2007: Effect of mesoscale eddies on Subtropical Mode Water variability from the Kuroshio Extension System Study (KESS). J. Phys. Oceanogr., 37, 982 1000, https://doi.org/10.1175/JPO3097.1.

Schneider, T., I. M. Held, and S. T. Garner, 2003: Boundary effects in potential vorticity dynamics. J. Atmos. Sci., 60 , 1024-1040, https://doi.org/10.1175/1520-0469(2003)60<1024: BEIPVD $>2.0 . \mathrm{CO} ; 2$.

Shchepetkin, A. F., and J. C. McWilliams, 2005: The regional oceanic modeling system (ROMS): A split-explicit, free-surface, topography-following-coordinate oceanic model. Ocean Modell., 9, 347-404, https://doi.org/10.1016/j.ocemod.2004.08.002.

Shcherbina, A. Y., E. A. D'Asaro, C. M. Lee, J. M. Klymak, M. J. Molemaker, and J. C. McWilliams, 2013: Statistics of vertical vorticity, divergence, and strain in a developed submesoscale turbulence field: Submesoscale turbulence statistics. Geophys. Res. Lett., 40, 4706-4711, https://doi.org/10.1002/grl.50919.

Skyllingstad, E. D., J. Duncombe, and R. M. Samelson, 2017: Baroclinic frontal instabilities and turbulent mixing in the surface boundary layer. Part II: Forced simulations. J. Phys. Oceanogr., 47, 2429-2454, https://doi.org/10.1175/JPO-D-16-0179.1.

Sullivan, P. P., and J. C. McWilliams, 2018: Frontogenesis and frontal arrest of a dense filament in the oceanic surface boundary layer. J. Fluid Mech., 837, 341-380, https://doi.org/ 10.1017/jfm.2017.833.

Taylor, J. R., and R. Ferrari, 2010: Buoyancy and wind-driven convection at mixed layer density fronts. J. Phys. Oceanogr., 40, 1222-1242, https://doi.org/10.1175/2010JPO4365.1.

Thomas, L. N., and P. B. Rhines, 2002: Nonlinear stratified spinup. J. Fluid Mech., 473, 211-244, https://doi.org/10.1017/ S0022112002002367.

— , and C. M. Lee, 2005: Intensification of ocean fronts by downfront winds. J. Phys. Oceanogr., 35, 1086-1102, https://doi.org/ 10.1175/JPO2737.1.

— , and R. Ferrari, 2008: Friction, frontogenesis, and the stratification of the surface mixed layer. J. Phys. Oceanogr., 38, 2501-2518, https://doi.org/10.1175/2008JPO3797.1.

—_ and J. R. Taylor, 2010: Reduction of the usable wind-work on the general circulation by forced symmetric instability. Geophys. Res. Lett., 37, L18606, https://doi.org/10.1029/ 2010 GL044680.

—, A. Tandon, and A. Mahadevan, 2008: Submesoscale processes and dynamics. Ocean Modeling in an Eddying Regime, Geophys. Monogr., Vol. 177, Amer. Geophys. Union, 17-38, https://doi.org/10.1029/177GM04.

Thompson, L., 2000: Ekman layers and two-dimensional frontogenesis in the upper ocean. J. Geophys. Res., 105, 6437-6451, https://doi.org/10.1029/1999JC900336.

Thorpe, A. S., and R. Rotunno, 1989: Nonlinear aspects of symmetric instability. J. Atmos. Sci. 46, 1285-1299, https://doi.org/10.1175/ 1520-0469(1989)046<1285:NAOSI >2.0.CO;2.

Tsuchiya, M., and L. Talley, 1998: A Pacific hydrographic section at $88^{\circ} \mathrm{W}$ : Water-property distribution. J. Geophys. Res., 103, 12 899-12 918, https://doi.org/10.1029/97JC03415.

Wenegrat, J. O., and M. J. McPhaden, 2016: Wind, waves, and fronts: Frictional effects in a generalized Ekman model. J. Phys. Oceanogr., 46, 371-394, https://doi.org/10.1175/ JPO-D-15-0162.1.

, and L. N. Thomas, 2017: Ekman transport in balanced currents with curvature. J. Phys. Oceanogr., 47, 1189-1203, https://doi.org/10.1175/JPO-D-16-0239.1.

Wong, A. P. S., and G. C. Johnson, 2003: South Pacific Eastern Subtropical Mode Water. J. Phys. Oceanogr., 33, 14931509, https://doi.org/10.1175/1520-0485(2003)033<1493: SPESMW $>2.0 . \mathrm{CO} ; 2$.

Young, W. R., 1994: The subinertial mixed layer approximation. J. Phys. Oceanogr., 24, 1812-1826, https://doi.org/10.1175/ 1520-0485(1994)024<1812:TSMLA > 2.0.CO;2. 